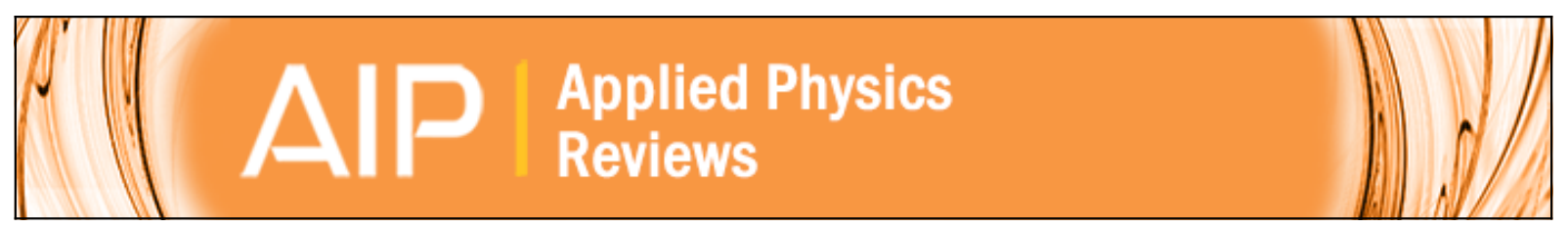

\title{
Pathways to exotic metastable silicon allotropes
}

Bianca Haberl, Timothy A. Strobel, and Jodie E. Bradby

Citation: Applied Physics Reviews 3, 040808 (2016); doi: 10.1063/1.4962984

View online: http://dx.doi.org/10.1063/1.4962984

View Table of Contents: http://scitation.aip.org/content/aip/journal/apr2/3/4?ver=pdfcov

Published by the AIP Publishing

\section{Articles you may be interested in}

Wide band gap carbon allotropes: Inspired by zeolite-nets

J. Appl. Phys. 120, 165101 (2016); 10.1063/1.4965721

Novel silicon allotropes: Stability, mechanical, and electronic properties

J. Appl. Phys. 118, 185704 (2015); 10.1063/1.4935549

Thermodynamic model for water and high-pressure ices up to $2.2 \mathrm{GPa}$ and down to the metastable domain J. Chem. Phys. 127, 124506 (2007); 10.1063/1.2768957

Polymorphism and metastable phenomena in liquid tin under pressure

Appl. Phys. Lett. 89, 221912 (2006); 10.1063/1.2397568

Light-induced metastability in hydrogenated nanocrystalline silicon solar cells

Appl. Phys. Lett. 85, 1925 (2004); 10.1063/1.1790072

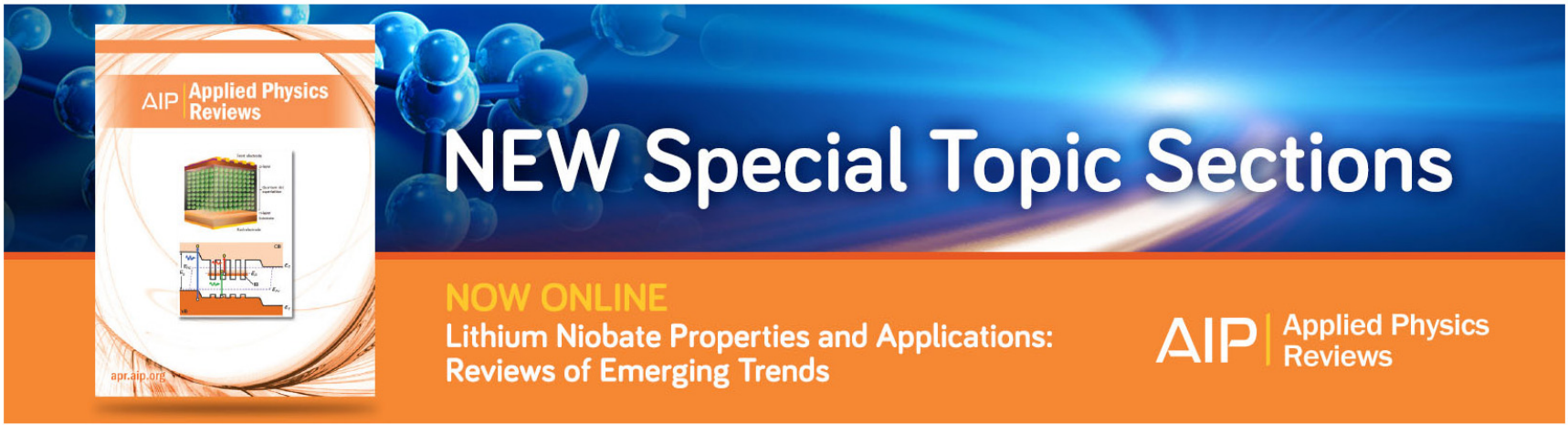




\title{
Pathways to exotic metastable silicon allotropes
}

\author{
Bianca Haberl, ${ }^{1}$ Timothy A. Strobel, ${ }^{2}$ and Jodie E. Bradby ${ }^{3}$ \\ ${ }^{1}$ Chemical and Engineering Materials Division, Neutron Sciences Directorate, \\ Oak Ridge National Laboratory, Oak Ridge, Tennessee 37831, USA \\ ${ }^{2}$ Geophysical Laboratory, Carnegie Institution of Washington, Washington, DC 20015, USA \\ ${ }^{3}$ Department of Electronic Materials Engineering, Research School of Physics and Engineering, \\ The Australian National University, Canberra 0200, Australia
}

(Received 28 March 2016; accepted 1 July 2016; published online 27 September 2016)

\begin{abstract}
The Group 14 element silicon possesses a complex free-energy landscape with many (local) minima, allowing for the formation of a variety of unusual structures, some of which may be stabilized at ambient conditions. Such exotic silicon allotropes represent a significant opportunity to address the ever-increasing demand for novel materials with tailored functionality since these exotic forms are expected to exhibit superlative properties including optimized band gaps for solar power conversion. The application of pressure is a well-recognized and uniquely powerful method to access exotic states of silicon since it promotes large changes to atomic bonding. Conventional high-pressure syntheses, however, lack the capability to access many of these local minima and only four forms of exotic silicon allotropes have been recovered over the last 50 years. However, more recently, significant advances in high pressure methodologies and the use of novel precursor materials have yielded at least three more recoverable exotic Si structures. This review aims to give an overview of these innovative methods of high-pressure application and precursor selection and the recent discoveries of new Si allotropes. The background context of the conventional pressure methods and multitude of predicted new phases are also provided. This review also offers a perspective for possible access to many further exotic functional allotropes not only of silicon but also of other materials, in a technologically feasible manner. Published by AIP Publishing. [http://dx.doi.org/10.1063/1.4962984]
\end{abstract}

\section{TABLE OF CONTENTS}

I. INTRODUCTION $\ldots \ldots \ldots \ldots \ldots \ldots \ldots \ldots \ldots$

A. Most exotic silicon phases come from static decompression .....................

B. Many new forms of silicon are plausible ...

II. UNCONVENTIONAL SYNTHESIS METHODS

FOR METASTABLE PHASES . .............

A. Synthesis via point loading ..............

1. Kinetic differences between indentation and DAC (de-)compression ............

B. Synthesis far away from equilibrium.......

1. Fs-laser shock compression in a confined geometry-Laser-induced microexplosions .................

C. Synthesis from metastable precursors ......

1. Topochemical and colloidal methods....

2. Framework-based and high-pressure precursors ......................

III. PERSPECTIVES FOR THE FIELD $\ldots \ldots \ldots \ldots$

A. Accessing additional functional allotropes ..

B. Scale-up to technologically interesting levels .........................

C. Transferability to other materials .......... IV. CLOSING REMARKS..................

\section{INTRODUCTION}

Our economy consistently pushes for the development of increasingly complex materials to meet the demands of high-efficiency energy conversion and high-performance

2 engineering challenges. Many new functional materials have

3 been predicted: materials optimized for solar power conversion, ${ }^{1-4}$ materials that exhibit room temperature superconductivity, ${ }^{5}$ and even materials with hardness higher than diamond. ${ }^{6,7}$ Syntheses of these revolutionary materials can only be met through innovative approaches. The application of extreme conditions provides a uniquely powerful and unprecedented opportunity to access such novel synthesis pathways: extreme conditions, in particular, pressure, enable access to new exotic states of matter located away from equilibrium of the elemental free energy landscape at standard conditions. Local minima within this energy landscape can then allow for metastable recovery and hence exploitation of such novel states of matter.

Given the ubiquitous nature of silicon, searches for such local minima in its free energy landscape (with the subsequent aim for metastable recovery of novel functional states) promise to be especially advantageous: Si lies at the heart of our modern technology as the material for integrated circuits $^{8,9}$ and also solar cells. ${ }^{10,11}$ Many applications are, however, limited since the band gap cannot be tuned easily 
and remains indirect. Hence, often other semiconductors are used, such as the expensive, highly toxic gallium arsenide for high-efficiency solar cells. ${ }^{12}$ Clearly, the synthesis of new, exotic structures that exhibit the desirable properties found in other materials, but maintain the earth-abundant, inexpensive, and non-toxic attributes of $\mathrm{Si}$ is extremely sought after.

The energy landscape of silicon is rich in (local) minima, i.e., the Group 14 element Si exhibits a very strong polymorphism and many different crystalline structures form under pressure, see, for example, Refs. 13-15 and those therein. Several metastable crystalline (and also amorphous) polymorphs have been recovered to ambient conditions already suggesting that additional structures may also be kinetically stable at ambient conditions. In the search for such structures, metastable and recoverable to ambient conditions, many highly useful allotropes of $\mathrm{Si}$ have been predicted (see below), especially allotropes useful for photovoltaics.

Their synthesis, however, is often unsuccessful by using standard high-pressure devices (such as diamond anvil cells (DACs)) because the application of extreme conditions to crystalline precursors in static pressure conditions is usually insufficient to overcome very strong kinetic barriers. This issue can be addressed if different avenues for the application of high pressure as well as novel precursor materials are exploited. Very recent work in this area does indeed demonstrate remarkable success of such approaches and several new functional $\mathrm{Si}$ allotropes have been recovered metastably to ambient conditions. ${ }^{16,17}$ The limitation of these novel approaches is entirely unknown and realization of their full potential promises access to further functional allotropes.

While we emphasize pressure and its role in providing access to these metastable states, it is just one of the many tools that might allow the practical realization of a second renaissance for element fourteen. Once new materials are discovered, novel precursor routes, soft chemical methods, or epitaxial deposition techniques could potentially enable scalability to industrially relevant levels.

The aim of this review is to elucidate these exciting new possibilities and avenues for the synthesis of novel functional states of $\mathrm{Si}$. To provide context, the common metastable structures recovered through static compression as well as the plethora of predicted allotropes are reviewed first in Sections I A and IB, respectively. This is followed by a detailed overview of the novel synthesis capabilities via point loading, laser-induced microexplosions, and metastable precursors in Section II. The future opportunities and possibilities for functional materials synthesis via high pressure as well as technological scalability will be discussed in Section III, before final concluding remarks are presented.

\section{A. Most exotic silicon phases come from static decompression}

Before proceeding, we note that naming conventions for structures vary widely throughout the literature. Hence, we attempt to provide colloquial names, space group information, Pearson symbols, and the three-letter codes (e.g., xyz), recommended by O'Keeffe and coworkers ${ }^{18}$ (see http://rcsr.net/nets) or the International Zeolite Association (XYZ), when available. We also make the point that many of these structures are found in other, related, chemical systems.

Conventionally, syntheses of metastable Si phases occur by applying pressure with a diamond anvil cell (DAC) to standard diamond-cubic Si (DC-Si, $F d \overline{3} m, c F 8$ dia) ${ }^{19}$ or amorphous $\mathrm{Si}(\mathrm{a}-\mathrm{Si}$ ) precursors to induce their metallization.

Thereby, a DAC consists of two opposing flat diamond anvils that are pushed together to apply extreme pressure to a small (in the order of a thousandth of a cubic mm) volume of material held in place by a gasket, e.g., Refs. 20 and 21 and references therein. The compression is normally conducted in a series of discrete loading or unloading steps at a timescale in the order of several minutes up to many hours or days. The structure of the resultant material throughout the loading and unloading process is usually measured directly via in situ X-ray diffraction or also via optical techniques such as Raman, infrared, or Brillouin spectroscopy. Recent work using gas membranes systems has enabled loading/ unloading and in situ $\mathrm{X}$-ray measurements to occur at a rate of several $\mathrm{GPa} / \mathrm{s}^{22}$

The metallization of silicon and its mechanisms have been reviewed in depth elsewhere, ${ }^{13-15}$ thus only a brief overview of the metallic phases is summarized here.

Under compression to $\sim 11 \mathrm{GPa}$, the tetrahedral DC-Si undergoes metallization into a structure analogous to white tin, $\beta$-Sn $\left(I 4_{1} /\right.$ amd, tI4) ${ }^{23}$ This metallic polymorph was initially named $\mathrm{Si}-\mathrm{II}$ but is now often described as $(\beta-\mathrm{Sn})-\mathrm{Si}$. During this metallization, the 4-fold coordinated semiconducting DC-Si becomes 6-fold coordinated through a considerable change in density of over $20 \%$. Above $\sim 13 \mathrm{GPa},(\beta-\mathrm{Sn})-\mathrm{Si}$ is distorted to the orthorhombic Imma structure (Si-XI. oI4). ${ }^{24,25}$ This polymorph transforms completely into an 8-fold coordinated simple hexagonal structure $(P 6 / \mathrm{mmm}, h P 1, \mathrm{Si}-\mathrm{V})$ at $15.4 \mathrm{GPa}^{24,26}$ Above $\sim 38 \mathrm{GPa}$, another orthorhombic structure (Si-VI, Cmca, oC16) forms, ${ }^{27}$ one that exhibits mixed 10 - and 11-fold coordination. Further compression to $\sim 42 \mathrm{GPa}$ yields the 12-fold coordinated hexagonal closepacked structure (space group $\left.P 6_{3} / \mathrm{mmm}, h P 2, \mathrm{Si}-\mathrm{VII}\right){ }^{26,27}$ Finally, at $\sim 79 \mathrm{GPa}$, the face-centered cubic structure (Si-X, $F m \overline{3} m, c F 4)^{28}$ is found, which is stable up to $248 \mathrm{GPa}$, the highest pressure probed to date using a DAC.

Although these metal-metal transitions are reversible on standard (i.e., linear, not rapid) decompression, the transition from DC-Si to $(\beta-\mathrm{Sn})-\mathrm{Si}$ is not and instead other 4-fold coordinated, but metastable phases are recovered. In 1962, Minomura and Drickamer ${ }^{29}$ noted a decreased resistivity of Si by following full decompression compared with the starting DC-Si. In 1963, Wentorf and Kasper ${ }^{30}$ identified the metastable phase formed upon full decompression as a bodycentered structure with 8 atoms per unit cell (space group $I a \overline{3}, t I 16$, gsi) as shown in Fig. 1 This phase, then called SiIII, is here described as $\mathrm{BC} 8$ based on its primitive structure. The structure was found to possess a $9 \%$ increased density compared with DC-Si and is expected to be kinetically stable "indefinitely" at ambient conditions.

Upon thermal annealing to $200{ }^{\circ} \mathrm{C}$, Wentorf observed the formation of a second metastable phase, HD-Si (also referred to as hex-Si, lonsdaleite $\mathrm{Si}$ or $\mathrm{Si}-\mathrm{IV}$ ) for its hexagonal 

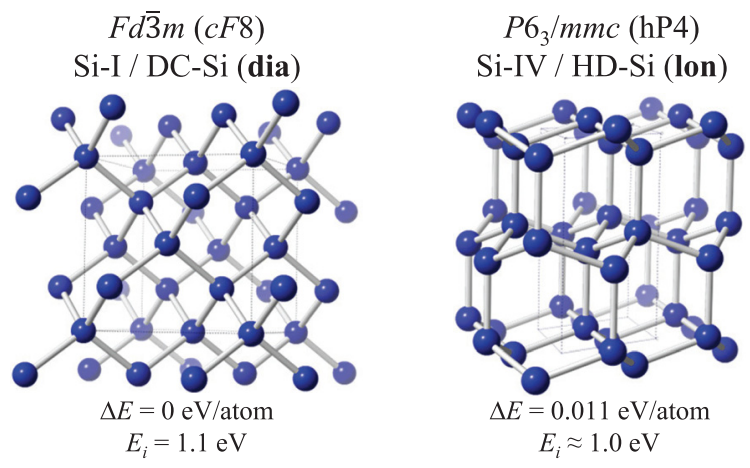

$\operatorname{Ia} \overline{3}(c I 16)$ $\mathrm{Si}-\mathrm{III} / \mathrm{BC} 8$ (gsi)

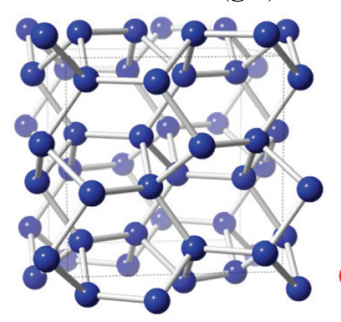

$\Delta E=0.159 \mathrm{eV} /$ atom

$E_{d} \approx 0.4 \mathrm{eV} /$ semimetal

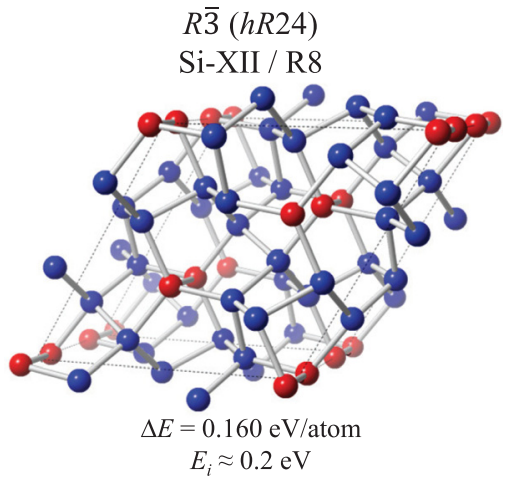

FIG. 1. Silicon allotropes stabilized at ambient conditions including DC-Si and the most common metastable phases (HD-Si, BC8-Si, and R8-Si) accessed through decompression from static pressure application. Formation energies relative to DC-Si are indicated, as well as direct $\left(\mathrm{E}_{\mathrm{d}}\right)$ or indirect $\left(\mathrm{E}_{\mathrm{i}}\right)$ band gaps. ${ }^{2,31-35}$ Different color atoms indicate unique crystallographic positions.

diamond structure. ${ }^{30}$ This phase possesses a hexagonal unit cell with 4 atoms, indeed the same structure as lonsdaleite/ hexagonal diamond $\left(\mathrm{Pb}_{3} / \mathrm{mmc}, h P 4\right.$, lon $)$, and is the monoatomic analogue of the wurtzite structure. This allotrope is also shown in as shown in Fig. 1. Consequently, it exhibits the same tetrahedral bond-length and -angles as DC-Si and thus also the same density.

The development of synchrotron radiation for in situ high-pressure studies enabled the discovery of three further metastable structures. In 1986, Zhao et al. observed that upon rapid unloading from 13 and $15 \mathrm{GPa}$, respectively, two new tetragonal phases formed. ${ }^{36}$ Since their full structures is unresolved, they remain described as "Si-VIII" and "Si-XI" only. In 1994, Crain et al. discovered an intermediate phase between the metallic $\mathrm{Si}$ and the final metastable BC8 structure. ${ }^{37}$ This rhombohedral phase also possesses 8 atoms in its unit cell $(R \overline{3}, h R 24)$ as shown in Fig. 1 . The structure represents a simple distortion of the BC8 phase and, initially called "Si-XII," is now often referred to as R8-Si. Although only traces of R8-Si remain after full decompression in a DAC, static decompression via point loading/indentation suggests a larger portion of remaining $\mathrm{R} 8-\mathrm{Si}$ beneath final indent impressions. ${ }^{38}$ This is discussed in more depth in Sec. II A.

Although the BC8-, HD-, and R8-Si (to some extent) polymorphs are largely recoverable at ambient conditions and hence potentially useful, their properties remain somewhat poorly characterized. It is clear, however, that their optoelectronic properties and hence functionality differ from
DC-Si. For example, Weill et al. found that BC8-Si is semimetallic, while HD-Si possesses an indirect band gap similar to $\mathrm{DC}-\mathrm{Si}^{35}$ and the $\mathrm{BC} 8 / \mathrm{R} 8$ mix created by point loading/ indentation is a narrow band gap semiconductor that can be doped at room temperature. ${ }^{39}$

Recent $a b$ initio considerations of these polymorphs suggest highly attractive functionality. For example, R8-Si has been predicted to possess a higher carrier mobility and a significantly better overlap with the solar spectrum (and hence significantly improved absorption properties) compared with standard DC-Si. ${ }^{33,34}$ Similarly, although BC8-Si in bulk form is expected to be semimetallic, calculations suggest a large band gap (up to several eV) for BC8 nanoparticles. The hydrogen-terminated form of such a nanoparticle may enable multiple exciton generation for solar cells and thus transcend the Shockley-Queisser limit for efficiency of solar cells. ${ }^{40}$ Moreover, Rödl et al. examined the electronic structure and optical transitions of HD-Si (Lonsdaleite/wurtzite). ${ }^{41}$ The calculations indicate that HD-Si is an indirect gap semiconductor $(0.95 \mathrm{eV})$, but an optically forbidden direct gap exists at $1.63 \mathrm{eV}$. Biaxial tensile strains were found to enhance the optical absorption properties, and a transformation into a direct gap material was observed with tensile strains exceeding $\sim 4 \%$. Thus, all three of these known metastable polymorphs of Si promise greatly improved band gap characteristics for future photovoltaic or other optoelectronic applications.

Despite the recent advances in experimental capabilities, no new metastable $\mathrm{Si}$ phases have been recovered in a diamond anvil cell using standard DC-Si or a-Si as precursors since R8-Si was identified in 1994. However, the greatly increased interest in novel functional silicon allotropes has resulted in a number of theoretical investigations of the silicon energy landscape which drives the search for new experimental pathways. These have revealed a multitude of feasible $\mathrm{Si}$ structures with potentially highly useful functionality. These allotropes and their potential for recovery to ambient conditions (in terms of competitive energetics) are reviewed next.

\section{B. Many new forms of silicon are plausible}

Pressure is the natural variable to distinguish energetic favorability of structures with varying densities. At high pressure, silicon takes on numerous dense forms with increasing coordination (as was summarized above), while openframework, low-density forms are favored at low pressure. It is interesting to note that clathrate topologies (e.g., type II mtn and type I mep) are calculated to be thermodynamic ground states under hydrostatic tension (negative pressure). ${ }^{42,43}$ At any particular P,T condition, there will exist one thermodynamic ground state (e.g., DC-Si at standard conditions), yet multitudes of metastable forms will exhibit energetic feasibility (i.e., favorable energetics not far from the ground state making them potentially kinetically stable at ambient conditions). Thousands of such energetically competitive, low-density structures have been computed, and numerous high-density forms are reasonable at elevated pressure. A subset of these energetically plausible structures exhibits well-behaved phonons (i.e., mechanical stability) 
and, of this subset, another offers promise for exciting physical properties and functionality. Indeed, there has been a recent explosion in the number of calculations on hypothetical silicon allotropes, largely driven by the desire to obtain new forms with enhanced optical properties. ${ }^{44}$

Given this surge in computational attention, one might ask: how many silicon allotropes could potentially be stabilized at ambient conditions? From the perspective of topology, there is no fundamental limit to the number of unique structures in the absence of constraints on symmetry, and the number of possible structures increases exponentially with the number of crystallographically distinct atoms. We might first constrain the situation to structure with four-coordinated atoms, which is the most likely scenario for $\mathrm{Si}$ at low pressure where $\mathrm{sp}^{3}$ forms dominate. Aside from the case of silicon, the mathematical enumeration of three-dimensional nets is an intriguing problem on its own (see, for example, Refs. 45 and 46). On the basis of graph theory, Delgado-Friedrichs et al. have enumerated $\sim 4 \times 10^{4}$ structure types for the case of four-coordinated nets with one unique vertex (think of a vertex as a unique crystallographic Wyckoff position), while $>4.4 \times 10^{6}$ types were obtained with two unique vertices. ${ }^{47}$ As pointed out by Zwijnenburg and coworkers, ${ }^{48}$ who calculated energies for many of these structure types with silicon atoms, most will exhibit prohibitively large energies with regards to practically realizing their existence, and thus energetics must also be considered.

From a theoretical standpoint, strategies to identify feasible silicon allotropes have largely revolved around analogy with known structures, global optimization methods (e.g., structure searching), or some combination of the two. The tetrahedral nature of silicon warrants consideration of isostructural silicates, and, indeed, zeolite-type structures have been considered broadly (as also done for carbon, e.g., Ref. 49 where $\mathrm{SiO}_{2}$ units were replaced by $\mathrm{C}$ atoms). Many zeolite structures exhibit favorable formation energies, including type I and II (mep and $\mathbf{m t n}$ ) clathrates, as well as many others (Fig. 2). While doped silicon clathrates have been known since the 1960s (and even ones with low alkali content) ${ }^{50}$ Adams et al. were the first to consider completely empty mep $(P m \overline{3} n, c P 46)$ and $\mathbf{m t n}(F d \overline{3} m, c F 136)$ forms of $\mathrm{Si}$ and calculated total energies $\sim 0.07 \mathrm{eV} /$ atom greater than DC-Si with wide band gaps near $2 \mathrm{eV} .{ }^{51}$ Demkov et al. expanded this work to additional zeolite structures including mtn, mep, bik, TON, cas, MTT, FER, sod, lta, fau, and even quartz. ${ }^{52}$ Many of these structures were found to have energies $\sim 0.1 \mathrm{eV} /$ atom greater than the diamond phase with band gaps $\pm 0.7 \mathrm{eV}$ from that of DC-Si. Several electronic structure calculations were subsequently performed on clathrate-type silicon, with the consensus that type I and type II have direct or quasidirect gaps (formally indirect, but with a very small energy difference between the direct gap) near $2 \mathrm{eV} .^{53-56}$

In an attempt to understand the structures of so-called allo-Si and $\mathrm{Ge}$, Conesa calculated several low-energy, lowdensity forms including mtn, mep, unj (called NGS), cas, and TON. ${ }^{57}$ Structural diversity in this low-density space was greatly expanded by Zwijnenburg et al. ${ }^{48}$ who calculated hundreds of structures with formation energies of $<0.35 \mathrm{eV} /$ atom. Karttunen et al. performed a comprehensive theoretical investigation of group 14 clathrate structures including polytypes, intergrowth frameworks, and extended structures using larger icosahedral building blocks. ${ }^{58}$ Karttunen et al. investigated more than 20 structures, and for
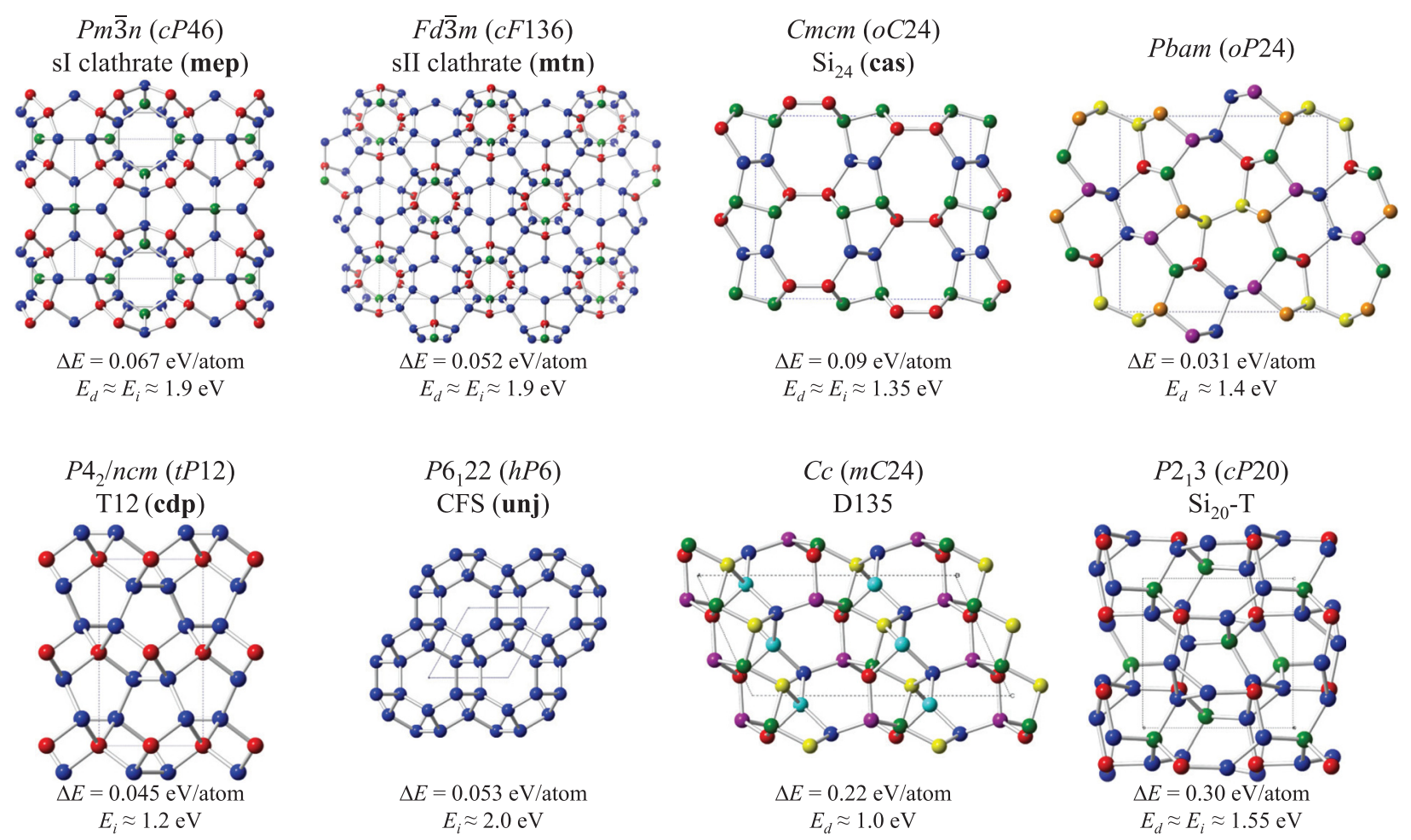

FIG. 2. Selected low-energy silicon allotropes. Formation energies at 1 atm relative to DC-Si are indicated, as well as direct $\left(\mathrm{E}_{\mathrm{d}}\right)$ or indirect $\left(\mathrm{E}_{\mathrm{i}}\right)$ band gaps. $^{2,16,51,52,55,56,67-69}$ Different color atoms indicate unique crystallographic positions. 
the case of silicon, all of them were found to be metastable within $0.3 \mathrm{eV} /$ atom relative to $\mathrm{DC}-\mathrm{Si}$. Many of the silicon structures were found to possess wide direct band gaps.

A series of orthorhombic structures, containing up to 32 atoms per cell, were proposed by $\mathrm{Chadi}^{59}$ based on the stacking of a two-dimensional ordered slab found within the structure of allo-Ge. ${ }^{60}$ The most stable structure contained 20 atoms (O-20) and was calculated to be metastable with respect to DC-Si by $0.03 \mathrm{eV} /$ atom with a $1.3 \%$ lower density. After applying symmetry to the translation vectors and atomic coordinates, the O-20 structure can be described in space group Pmma (oP20) and appears to be the same structure found by Baburin et al. for carbon. ${ }^{61}$

In 1991, Boyer et al. ${ }^{62}$ predicted a body-centered tetragonal structure (BCT-5; I4/mmm, tI4), sqp) with fivefold coordination to be energetically competitive with the ( $\beta$-Sn)-Si at pressures near $10 \mathrm{GPa}$. This phase was constructed by displacing atoms within the DC-Si structure and a strain-induced pathway was proposed for its synthesis. In 2012, Gerbig et al. suggested the potential synthesis of BCT-5 based on Raman observations in nanoindentation experiments. ${ }^{63}$

Galvani et al. ${ }^{64}$ proposed a hexagonal "nanoporous" silicon crystal (previously predicted for carbon ${ }^{65}$ ) with 40 atoms per unit cell (Hex Si-40). The structure was calculated to have a bandgap of $>0.4 \mathrm{eV}$ beyond that of DC-Si (i.e., $\sim 1.5 \mathrm{eV}$ ), with energetic stability comparable to type-I (mep) and type-II (mtn) clathrates. This $P 6 / \mathrm{mmm}$ structure (type-III clathrate, zra-d) is constructed by tiling related polyhedral cages together $\left(2\left[5^{12} 6^{3}\right]+2\left[5^{12} 6^{2}\right]+3\left[5^{12}\right]\right)$ and was also calculated by O'Keefe and coworkers, ${ }^{66}$ by Zwijnenburg et al., ${ }^{48}$ and by Karttunen et al. ${ }^{58}$

Another BCT silicon phase was proposed by Fujimoto et al. in $2008 .^{70}$ This BCT phase, also with $14 / \mathrm{mmm}$ ( $\left.t I 8\right)$ symmetry, was calculated to possess an indirect band gap of $0.47 \mathrm{eV}$ using the GW approximation method. This phase has the crb topology and was calculated previously for carbon [the reader is encouraged to examine the so-called Samara Carbon Allotrope Database (SACADA) $\left.)^{71}\right]^{72}$ This BCT Si was further calculated by Malone, Louie, and Cohen who determined an indirect gap of $0.86 \mathrm{eV}$ using the GW approximation method and attributed possible differences in the gap magnitude to differences in plane-wave versus augmented-wave implementations. ${ }^{73}$

In 2010, Pickard and Needs rediscovered the NGS/unj silicon structure $\left(P 6_{1} 22, h P 6\right)$, first calculated by Conesa ${ }^{57}$ for silicon, and listed by $\mathrm{O}^{\prime}$ Keefe and Brese, ${ }^{74}$ calling it a chiral framework structure (CFS). ${ }^{67}$ They suggest metastability of $0.053 \mathrm{eV} /$ atom relative to $\mathrm{DC}-\mathrm{Si}$, which is similar to the findings of Conesa. The band gap was found to be indirect and calculated to have a magnitude of $1.46 \mathrm{eV}$ using density functional theory (DFT) with the generalized gradient approximation (GGA). Using the same method, a band gap of $0.6 \mathrm{eV}$ was reported for DC-Si (so, the actual gap of NGS/unj is likely $\sim 2 \mathrm{eV}$ ).

Botti et al. ${ }^{1}$ performed calculations for approximately 300 semiconducting silicon allotropes that were previously identified for carbon ${ }^{75}$ using the minima hopping method. ${ }^{76}$ Of these, 16 structures were identified to have energies of $\leq 0.15 \mathrm{eV} /$ atom above DC-Si. While none of the structures were found to have formally direct band gaps, many of them displayed quasidirect gaps and displayed improved visible light absorption. Note that the "Cco-Si ${ }_{8}$ " structure calculated by Zhai et al. ${ }^{77}$ during the same time period is the same structure as " $Z$ " silicon and carbon (and also the same as "Cco- $\mathrm{C}_{8}$ "78 and (RL)2 ${ }^{79}$ ). "M" (cbn) and " $\mathrm{Z}$ " (sie) silicon were also calculated by Bautistia-Hernandez et al. ${ }^{80}$ in 2013.

Zhao et al. $^{81}$ found a tetragonal allotrope with 12 atoms per cell ("T12," (cdp), $P 4_{2} / \mathrm{hcm}$ ) using the CALYPSO structure searching method. ${ }^{82}$ This phase was calculated to be very energetically competitive $(<0.05 \mathrm{eV} /$ atom $)$ using DFT with the local density approximation (LDA). Interestingly, the energy barrier from DC-Si to T12 was calculated to be similar compared with DC-Si to $\mathrm{R} 8 / \mathrm{BC} 8$, and calculated Raman features for this phase were thought to explain observations from $\mathrm{Si}-\mathrm{XIII}$, which structure is still unresolved. ${ }^{83}$

Several silicon allotropes based on possible solutions to the Kelvin Problem (how to partition 3D space into cells of equal volume with minimal surface area) were uncovered by Zhao et al. in 2013. ${ }^{84}$ These are all semiconducting with GGA-based DFT gaps between 0.2 and $1.4 \mathrm{eV}$ and formation energies below $\sim 0.2 \mathrm{eV} /$ atom.

Xiang et al. predicted a " $\mathrm{Si}_{20}-\mathrm{T}$ " phase with space group $P 2{ }_{1} 3$ (cP20). ${ }^{69}$ The structure was uncovered by searching for direct band gap phases using particle swarm optimization combined with an inverse band structure approach. $\mathrm{Si}_{20}-\mathrm{T}$ was calculated to possess a quasidirect gap of $1.55 \mathrm{eV}$ (using the hybrid functional HSE06) and is metastable to DC-Si by about $0.3 \mathrm{eV} /$ atom. The material was calculated to have significantly improved visible light absorption compared with DC-Si and was demonstrated to be mechanically stable and thermally stable in molecular dynamics simulations at $350 \mathrm{~K}$.

Six silicon allotropes with direct or quasidirect band gaps were calculated by Wang et al. in $2014 .^{85}$ The formation energies of all of them are $<0.3 \mathrm{eV} /$ atom and calculated band gaps ranged between $0.39 \mathrm{eV}$ and $1.39 \mathrm{eV}$ (HSE06). A few known topologies can be found for these structures. The Cmcm (oC12) phase is related to the bikitaite zeolite structure (bik) (calculated for silicon in Ref. 51 and for carbon in Ref. 49), the $I 4 / \mathrm{mcm}$ ( $t I 16$ ) phase is a framework based on $\mathrm{TlZn}_{2} \mathrm{Sb}_{2}$ (tzs), and the $P 6_{3} / m m c(h P 12)$ phase has the topology smv.

Nguyen et al. found another tetragonal allotrope via a genetic algorithm structure searching approach. ${ }^{86}$ The structure has the space group $P 4_{2} / \mathrm{mnm}(\mathrm{P} 12)$ and is the isq topology originally proposed by O'Keeffe. ${ }^{87,88}$ The formation enthalpy was calculated to be $0.113 \mathrm{eV} /$ atom and an indirect band gap of $1.28 \mathrm{eV}$ was determined (DFT GGA). This phase was investigated further in recent computations. ${ }^{89}$

Lee and coworkers used conformal space annealing with 10-20 silicon atoms per cell to screen for direct band gap crystals. ${ }^{68}$ The method produced a large number of lowsymmetry (mostly $P 1$ ) structures with competitive energies $(<0.3 \mathrm{eV} /$ atom $)$. Several structures were calculated with direct band gaps between 1 and $2 \mathrm{eV}$ using the GW approximation. This work was expanded to include global optimization with conformal space annealing to predict additional structures with direct band gaps. ${ }^{90}$ Six additional crystals 
were found with direct gaps near $1 \mathrm{eV}$ and formation energies of $<0.22 \mathrm{eV} /$ atom.

Amsler et al. performed a comprehensive search based on clathrate materials using the minima-hopping method. Fictitious Lennard-Jones (LJ) spheres were used to simulate guest atoms and bias low-density, clathrate-type structures. ${ }^{4}$ Known clathrates (e.g., mep, mtn) and Kelvin structures ${ }^{84}$ were used as starting points to create new structures with similar building blocks. After filtering thousands of initial structures, roughly 150 were considered for further analysis. Among those, several previously considered topologies were identified and some previously unconsidered silicon phases are related to other types of known tilings (e.g., the "C01" structure with the lowest formation enthalpy is related to the dual $\mathrm{NbBe}_{3}$ structure (nbd). ${ }^{91}$ Notably, eleven phases were predicted to possess direct or quasidirect bandgaps between 1 and $1.8 \mathrm{eV}$. Absorption profiles for selected structures are shown in Fig. 3.

The random structure search method ${ }^{92}$ was applied by Mujica et al. to predict several low-energy silicon allotropes, at both low and high densities. ${ }^{2}$ On the high-density side, the $I 4_{1} / a$ (tI16) structure was found to be nearly energetically degenerate with R8/BC8. This phase with a body-centered tetragonal structure with 16 atoms in its unit cell was previously predicted based on the considerations of the transition pathway from ( $\beta$-Sn)-Si to $\mathrm{BC} 8-\mathrm{Si}$ and analogously called BT8. ${ }^{93}$ Moreover, a $\mathrm{P6}_{4} 22$ ( $h P 20$, pseudocinnabar) structure was observed to have similar energy to the Ibam phase proposed by Malone and Cohen. ${ }^{94}$ At lower density, several new structures were found. Notably, Pbam (oP24) was calculated to have a direct band gap of $1.4 \mathrm{eV}$ and formation energy of only $0.029 \mathrm{eV} /$ atom. We note that this phase was also found for carbon by Baburin and coworkers. ${ }^{61}$ In addition, a $P 4_{1} 2{ }_{1} 2(t P 20)$ phase containing fivefold spirals of atoms was calculated to be metastable with respect to DC-Si by $0.041 \mathrm{eV} /$ atom and was suggested as a more likely candidate structure for Si-XIII than "T12" based on agreement with simulated Raman spectra.

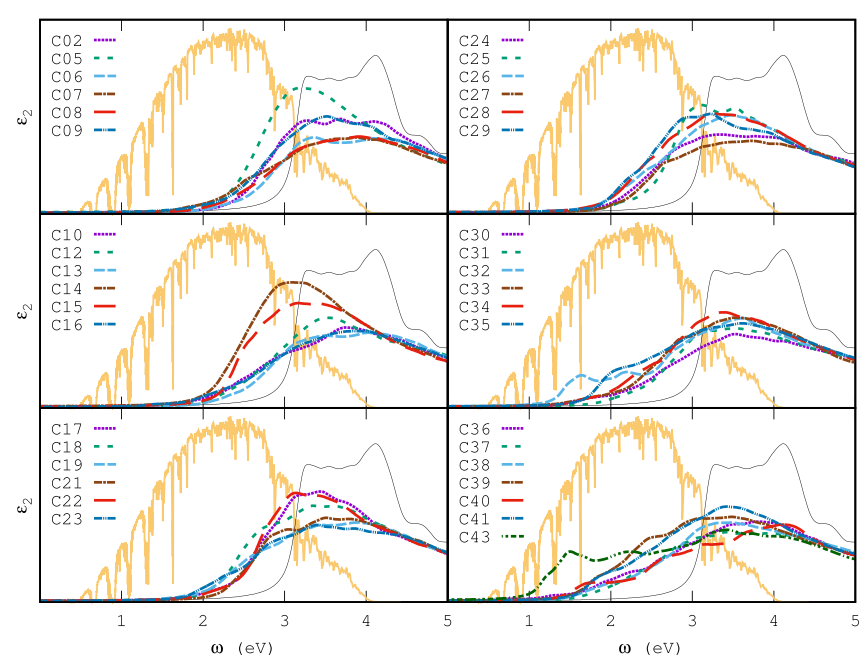

FIG. 3. Imaginary portion of the dielectric function $\left(\varepsilon_{2}\right)$ for selected lowdensity structures based on the work by Amsler et al. ${ }^{4}$ Solid black lines in each panel represent DC-Si and the yellow curves are the AM-1.5 solar spectrum.
A co-author of the above study identified several further higher-density allotropes with competitive energetics using the same approach. ${ }^{17}$ Two tetragonal structures with 32 atoms, i.e., two forms of "T32," were found, one with space group $P \overline{4} 2_{1} c$ (tP32) and $0.163 \mathrm{eV} /$ atom difference to DC-Si and one with space group $P 4_{3} 2_{1} 2(t P 32)$ and $0.164 \mathrm{eV} /$ atom difference to DC-Si. The same random structure searches also uncovered two monoclinic structures with 32 atoms: $C 2(m C 32)$ and $P 2_{1} / c$ (mP32) with energy differences from DC-Si of $0.162 \mathrm{eV} /$ atom and $0.163 \mathrm{eV} /$ atom, respectively.

Zhu et $\mathrm{al}^{3}{ }^{3}$ used an evolutionary metadynamics approach to independently find one of these low-energy "T32" allotropes $\left(P \overline{4} 2_{1} c, t P 32\right)$. This phase has many structural similarities with the BC8 and R8 structures, which can all be characterized by different arrangements of spiral chains. The structure was found from simulations starting from DC-Si/ $(\beta-\mathrm{Sn})-\mathrm{Si}$ and its formation energy of $0.164 \mathrm{eV} /$ atom is nearly equivalent to those of $\mathrm{BC} 8 / \mathrm{R} 8$. Calculations of the T32 band structure using HSE06 revealed a quasidirect gap of $1.28 \mathrm{eV}$.

Tang et al. predicted a direct gap allotrope $(\sim 1.6 \mathrm{eV})$ with a "nanotubular" structure $\left(I_{1} /\right.$ amd $) .{ }^{95}$ It is a lower density phase than DC-Si and metastable by $\sim 0.2 \mathrm{eV} /$ atom. The precise structural description is unclear, but the structure seems to be the same as LA10 (mon) predicted in Ref. 96.

Four additional semiconducting low-energy silicon phases were reported by Fan et al.: Amm2 (oA20), C2/m ( $m C 16), C 2 / m$ ( $m C 20)$, and $I \overline{4}(t I 28) .{ }^{97}$ All four structures were reported previously for carbon. ${ }^{98}$ The calculations at the GGA-based DFT level revealed that the Amm2 silicon phase has a quasidirect band gap of $0.742 \mathrm{eV}$ and a formation energy of $0.109 \mathrm{eV} /$ atom.

Guo et al. proposed a direct band gap $(0.61 \mathrm{eV}$ using HSE06) structure based on the tiling of triangular rings. ${ }^{99}$ This low-density "h-Si ${ }_{6}$ " structure $\left(P 6_{3} / m m c, h P 6\right)$ was calculated to be $0.35 \mathrm{eV} /$ atom higher in energy than DC-Si and was predicted to exhibit a large carrier mobility of $\sim 10^{4} \mathrm{~cm} / \mathrm{V} \cdot \mathrm{s}$. The open-framework structure has the same topology as sny. ${ }^{18}$

We also briefly note that a vast number of superstructures and stacking-derived polytypes (similar to those for carbon, see, for example, Ref. 100) are also possible and energetically feasible for silicon. We point the reader to refer Refs. 101-107 for more detailed discussions on this subject.

It is clear that the energy landscape of silicon is complex with multitudes of local minima and plausible metastable structures. But how can we actually access these states? We will next review three different methods to enable kinetic control of synthesis that provide possible pathways into the realms of these exotic forms of silicon: point loading, nonequilibrium synthesis, and metastable precursor methods.

\section{UNCONVENTIONAL SYNTHESIS METHODS FOR METASTABLE PHASES}

\section{A. Synthesis via point loading}

Indentation is a method of applying a localized pressure to a material via uniaxial point loading. The method is conceptually simple and is most commonly achieved via the use of instrumented-indentation systems that are primarily 
designed to measure mechanical properties such as hardness and Young's Modulus. ${ }^{108,109}$ Forces in the range of $\mu \mathrm{N}$ to $\mathrm{N}$ can be routinely applied while simultaneously the penetration depth of the indenter tip below the surface of the sample is measured. Indentation tips are made from diamond or other super-hard materials. The tips are generally spherical (diameters ranging from $100 \mathrm{~nm}$ to $1 \mathrm{~mm}$ ), flat-punches, or pyramidal in shape. The maximum pressure is primarily determined by the yield point of the tested material and as the tip contact areas are usually quite small (in the order of $1-10 \mu \mathrm{m}^{2}$ ), the magnitude of the applied force is not a limiting parameter in terms of reaching pressures in the $\mathrm{GPa}$ range. In the case of $\mathrm{DC}-\mathrm{Si}$, indentation induces pressures high enough to induce metallization $(\sim 11 \mathrm{GPa}) .{ }^{110-113}$ This is supported by analytical calculations, by in situ electrical measurements, and by ex situ analysis of the residual indent impressions where clear evidence of non-DC-Si phases has been observed using electron microscopy and Raman spectroscopy. ${ }^{114-117}$

The first evidence of an indentation-induced phase transformation in $\mathrm{Si}$ was obtained via the observation of a dramatic resistance change between metal contacts on DC-Si during indentation. ${ }^{117}$ The resistance was found to drop during loading suggesting metallization occurred under the indenter tip. This led Gerk and Tabor to speculate on the formation of $(\beta-\mathrm{Sn})-\mathrm{Si}$ under the indentation tip giving rise to plasticity in $\mathrm{Si}^{110}$ However, the direct observation of phase transformation induced via point loading was not seen until a number of years later when a region of amorphous $\mathrm{Si}$ in a residual indent impression formed by Vickers indentation was imaged by transmission electron microscopy. ${ }^{118}$ Although by this time (1988) it was relatively well understood from the DAC literature that Si undergoes a series of hysteretic pressure-induced phase transformations, the discovery of a-Si instead of crystalline $\mathrm{BC} 8-\mathrm{Si}$ as the residual structure was a surprising outcome. This remained essentially unexplained until the development of instrumented nanoindentation enabled a more detailed study of the deformation processes to be explored.

The continuous measurement of the load and depth revealed a number of features in both the loading and unloading segments of the indentation of $\mathrm{Si}^{111,112,119,120} \mathrm{~A}$ discontinuity, called a "pop-in" event, is seen when loading using a spherical indenter but is often absent when loading with a pointed (Berkovich) tip. ${ }^{112,115}$ In situ electrical measurements under indentation confirmed that a metallic phase is created during indentation loading ${ }^{111}$ and that incipient plasticity is responsible for the "pop-in" event during spherical tip loading. ${ }^{113}$ On unloading, however, two distinct behaviors are observed regardless of the indenter geometry. On slow unloading, a so-called "pop-out" event is seen, whilst an elbow is observed when faster unloading rates are used. Examples of this are shown in Fig. 4.

Subsequent analysis of the residual indent impression using Raman spectroscopy and electron microscopy reveals that the unloading rate controls the formation of the final structure. ${ }^{114,116,121}$ Amorphous Si is observed as the residual Si structure when "elbows" are seen in the unloading data, whilst a pop-out event results in a mixture of the $\mathrm{BC} 8 / \mathrm{R} 8$
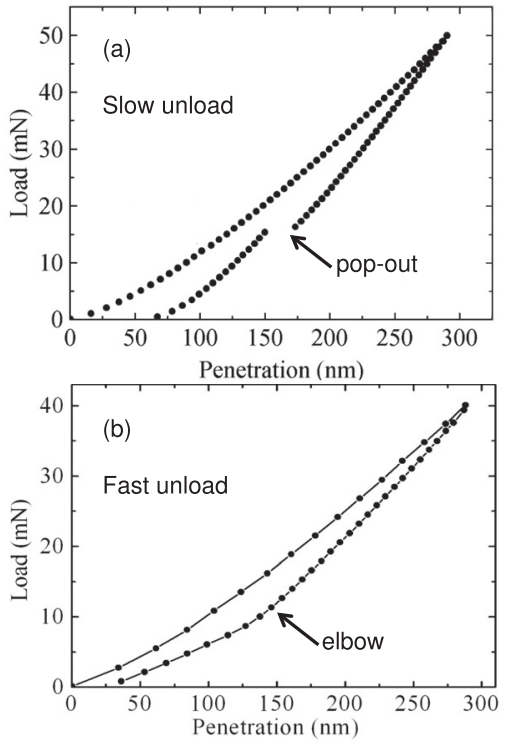

FIG. 4. Indentation load-unload curves of DC-Si using an $\sim 5.0 \mu$ m-radius spherical indenter with an unloading rate of (a) $\sim 3 \mathrm{mN} / \mathrm{s}$ and (b) $\sim 10 \mathrm{mN} / \mathrm{s}$.

structure $^{115,121}$ as shown in Fig. 5. These transmission electron microscopy images show that there is significant damage to the underlying crystal and that the diameter of the phase transformed region is limited to $\sim 2 \mu \mathrm{m}$. Very recent work with large-diameter $(20 \mu \mathrm{m})$ spherical tips has shown that it is possible to promote phase transformation as the sole mode of indentation-induced deformation in $\mathrm{DC}-\mathrm{Si}^{122}$ and
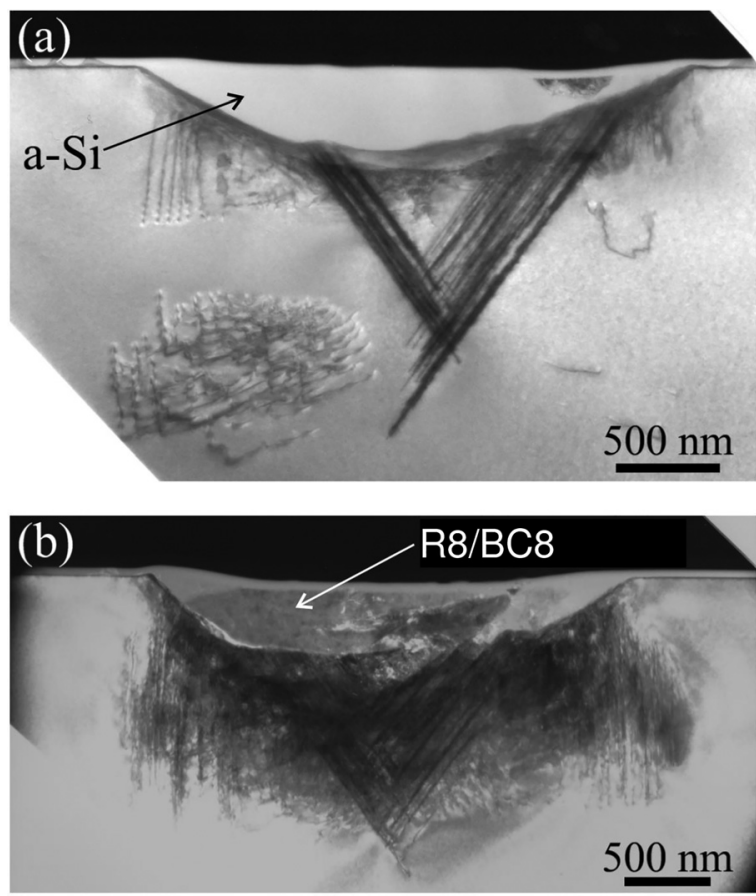

FIG. 5. Transmission electron microscopy images of residual indent impressions formed using a spherical indenter of radius $\sim 5 \mu \mathrm{m}$ loaded to $80 \mathrm{mN}$. (a) Final structure of amorphous Si after "fast" final unload $(\sim 10 \mathrm{mN} / \mathrm{s})$ and (b) final structure containing a mixture of R8/BC8-Si after "slow" unload $(\sim 3 \mathrm{mN} / \mathrm{s})$. This rate dependence shown here is adapted with permission from Bradby et al., J. Mater. Res. 16, 1500-1507 (2001). Copyright 2001 Materials Research Society. 
that the application of a hold period can promote the elasticplastic transition to lower loads. ${ }^{123}$

\section{Kinetic differences between indentation and DAC (de-)compression}

Although the transition sequences are similar in DAC experiments and the indentation (un-) loading detailed above, there are some distinct differences. First, unlike in a DAC, R8-Si is stabilized after indentation although it always appears alongside $\mathrm{BC} 8-\mathrm{Si}^{39}$ Note, these findings are largely based on Raman microspectroscopy performed on residual indent impressions which show a strong prominence of the main R8-Si band over the main BC8-band. ${ }^{116,124}$ Due to different Raman cross-sections of the semimetallic BC8-Si and the semiconducting $\mathrm{R} 8-\mathrm{Si}$, the exact $\mathrm{BC} 8$-to- $\mathrm{R} 8$ ratio remains unknown. Nevertheless, the R8/BC8 mixture that routinely is formed after indentation behaves electrically different to BC8. It has been shown to act as narrow band-gap semiconductor and is able to be doped at room temperature. ${ }^{39}$ This is an intriguing difference that has been attributed to remaining stress/strain in the residual indent impression of $\sim 1 \mathrm{GPa} .{ }^{124}$

Further differences between the DAC and the indentation results relate to the formation of the a-Si structure after fast indentation decompression. ${ }^{116}$ The current understanding of this behavior suggests that the rapid decompression from metallic ( $\beta$-Sn)-Si formed under the indentation tip prevents the nucleation of $\mathrm{BC} 8 / \mathrm{R} 8-\mathrm{Si}$ and instead the highenergy amorphous structure is formed. ${ }^{125}$ That is, the small phase transformed volumes present under indentation (decompression are nucleation limited. This appears fundamentally different to the kinetic pathways in a DAC, where fast decompression from $\left(\beta\right.$-Sn)-Si does not ever yield a-Si. ${ }^{126}$ The exact reason for these differing kinetic pathways is not understood and remains under investigation. It is an area of some interest since the pure a-Si formed by rapid indentation unloading is not structurally the same as other pure forms of a-Si. ${ }^{127,128}$ Detailed structural investigations suggest instead that it possesses an elevated density over other forms of a-Si and less structural order. ${ }^{127,128}$ This is clearly reminiscent of its formation pathway from a high-density, high-coordination metallic phase via a nucleation-limited rapid decompression pathway.

Another important example of kinetic differences between phases formed upon indentation and DAC decompression is the formation of Si-XIII. Si-XIII is a polymorph of $\mathrm{Si}$ with unidentified structure that forms upon thermal $\sim 200^{\circ} \mathrm{C}$ ex situ annealing of the $\mathrm{BC} 8 / \mathrm{R} 8$ mixture made by indentation. ${ }^{124,129,130}$ This phase has been detected by Raman microspectroscopy ${ }^{129}$ and its existence is now well established. ${ }^{124,130}$ It has, however, proved extremely difficult to identify its structure since the phase is not amenable to transmission electron microscopy studies ${ }^{130}$ and the volumes made by single indentation is not sufficient for $\mathrm{X}$-ray diffraction. Although this Si-XIII very clearly forms from BC8/R8$\mathrm{Si}$ it has been impossible to replicate this transformation pathway in a DAC, even though different $\mathrm{BC} 8 / \mathrm{R} 8$ ratios, stresses, and heating rates have been applied. ${ }^{130}$ Thus, the formation of Si-XIII is clearly unique to the particular BC8/ R8-Si created by indentation.

One possible explanation for the absence of Si-XIII in DAC experiments could lie in its density. Optical and electron microscopy imaging of phase transformed zones may suggest that Si-XIII exhibits a lower density than BC8/R8$\mathrm{Si}$. Thus, it is conceivable that it can form in the indentation case where the top surface of the phase transformed volume is entirely unconfined after full decompression. In contrast, in the DAC case, the diamond anvils maintain constant pressure-volume and would thus prevent the necessary expansion toward a low-density phase. Interestingly, if SiXIII should prove to be a low-density phase, it could well be related (or indeed be) the recently predicted low-density tetragonal $\mathrm{P} 4{ }_{1} 2{ }_{1} 2$ structure which does match the known Si-XIII Raman bands. ${ }^{2}$

In summary, it is clear that although indentation represents a case of static (de-)compression, different kinetic conditions to those present in a DAC can be accessed. This yields the "freezing-in" of a larger portion of the technically interesting R8-Si phase, the formation of a pure form of a-Si, and promotes the nucleation of an entirely new Si allotrope, Si-XIII.

\section{B. Synthesis far away from equilibrium}

Another method to access metastable structures far from equilibrium is the application of shock wave compression (see, for example, Refs. 131 and 132 and those therein). A shock wave propagates within a solid material at speeds faster than the speed of sound thus inducing significant compression at the front of the wave. Shock waves in a solid material can be induced via several methods, via interaction with a (very fast) moving impactor, ${ }^{131}$ via an explosive detonation, ${ }^{133}$ via a laser-shock event, ${ }^{132}$ or even via a lasershock within a static DAC. ${ }^{134}$ In the context of materials synthesis far from equilibrium, it is useful to distinguish between equilibrium and non-equilibrium shock conditions: Non-equilibrium conditions may be defined as conditions under which the energy given to the system cannot couple to the lattice, e.g., non-linear conditions in the femtosecond regime where, for example, a laser couples initially to the electrons only. In contrast, equilibrium conditions may be defined as linear, static pressure (and temperature) conditions such as in a DAC or under indentation. This also includes picosecond or nanosecond laser-application where the laser couples to the lattice instead of just the electrons.

The appropriateness of these definitions is somewhat reflected in the phase transitions that occur upon exposure of silicon to (laser-induced) shock. The only metastable phases reported from "equilibrium" shock exposure were metastable phases also recovered in a DAC (BC8, R8, Si-VIII) and amorphous silicon. In contrast, fs-laser compression in a confined geometry has very recently yielded additional metastable tetragonal structures (ST12, BT8). These findings are now reviewed in detail.

The first shock compression experiments on $\mathrm{Si}$ carried out by Al'tshuler and Pavlovskii utilized detonation explosives coupled with connectivity measurements. ${ }^{135,136}$ These 
measurements yielded metallization of $\mathrm{Si}$ under pressure, with an onset pressure of $10 \mathrm{GPa}$. Further shock compression experiments utilizing a flyer plate conducted by Goto et al. in 1982 reported metallization of $\mathrm{Si}$ albeit at the slightly elevated pressure of $13.4 \mathrm{GPa}^{137}$ A later comprehensive study by Gilev and Trubachev also utilized time-resolved conductivity measurements to determine the effects of shock on a $\mathrm{Si}$ plate loaded into a cell to-be shocked with two methods, via a plane wave generator or via a metallic plate accelerated via high explosives. ${ }^{138}$ This study was able to fully confine the phase transition pressure in $\mathrm{Si}$ under shock compression to an onset pressure of $10 \mathrm{GPa}$ and a completion pressure of $12 \mathrm{GPa}$, in excellent agreement with the static DAC findings.

On release, these shock studies reported a hysteresis in conductivity (somewhat akin to the transition to R8- and $\mathrm{BC} 8-\mathrm{Si}$ in DAC decompression). The detailed nature of the recovered material remains somewhat unclear, however. Even a series of ex situ shock-recovery experiments performed by Kishimura and Matsumoto did not resolve the question since no clear evidence for the formation of known metastable crystalline phases was detected. ${ }^{139}$

More information on the material recovery has been obtained from laser application. A large body of work focuses more on laser-melting than compression. Early work by Liu et al. found that laser-induced amorphization can result from the application of ps-laser pulses. ${ }^{140}$ Such amorphization was later confirmed also for the application of fs-lasers and thus for non-linear, non-equilibrium conditions. ${ }^{141,142}$ In this case, however, much ablation occurs and not much transformed material remains for study.

Nonetheless, over the last five years, a number of laserinduced shock studies did indeed report formation of metastable crystalline phases. Two different electron microscopy studies performed on recovered samples by Schade et al. and Smith et al. observed the formation of an amorphous layer on the ripple structures resulting from fs-laser irradiation. ${ }^{143,144}$ Accompanying Raman microspectroscopy detected additional evidence of the R8 phase, in the rim of the ablation crater in the former case and across the entire laser irradiated zone in some instances in the latter case. Furthermore, another fs-laser shock study by Tsujino et al. utilizing X-ray diffraction detected the presence of Si-VIII instead. ${ }^{145}$ The formation of these metastable crystalline phases was largely attributed to shock in addition to laser-melting.

It is highly noteworthy that despite the laser-induced shock being caused by non-linear fs-lasers, the same metastable structures are also observed in static DAC experiments. This may be attributed to the fact that all these fs-laser events occurred on the surface of the sample and are always accompanied by ablation. This appears to prevent the full utilization of non-equilibrium conditions. Instead, it is conceivable that these standard metastable crystalline structures formed via recovery from metallic $\mathrm{Si}$ melt [which is also 6-fold coordinated and similar in structure to $(\beta$-Sn)$\mathrm{Si}]^{146}$ in conjunction with the shock wave. Interestingly, a further study using ns-lasers (and thus equilibrium conditions) also reported the formation of $\mathrm{R} 8-\mathrm{Si}$ as detected by Raman microspectroscopy. ${ }^{147}$ In this case, an IR laser was used to enable a focal point well beneath the surface of the $\mathrm{Si}$. Thus, confinement appears to prevent any ablation and alters the laser-material interactions.

It summary it is clear that laser-induced shocks can result in phase transitions to metastable crystalline phases in Si provided that suitable conditions (such as fs-lasers or confinement) are chosen. Thus, the question arose whether it is possible to exploit such conditions in the search of further metastable structures that have not been recovered under static conditions yet. Clearly, a combination of fs-laser shocks and confining geometries is promising as detailed now.

\section{Fs-laser shock compression in a confined geometry-Laser-induced microexplosions}

True confinement of Si under fs-laser exposure can be realized through the use of thermally grown $\mathrm{SiO}_{2}$ as a surface layer. Such a layer is attached entirely voidless to the $\mathrm{Si}$ substrate and is transparent for laser-processing. Therefore, a laser beam can be focused directly onto the Si substrate in a confined manner, i.e., without ablation occurring, to induce highly non-equilibrium, non-linear conditions within the sample. ${ }^{17,148,149}$

In a recent study, a $170 \mathrm{fs}, 790 \mathrm{~nm}, 0.1-1.0 \mu \mathrm{J}$ single pulse laser was tightly focused with a $150 \times$ high numerical aperture objective lens $(\mathrm{NA}=1.45)$ onto the $\mathrm{Si}$ surface beneath the $\mathrm{SiO}_{2}$ layer to induce a highly localized laser event, a so-called laser-induced microexplosion. ${ }^{17}$ For $1 \mu \mathrm{J}$ focused into a $1 \mu \mathrm{m}^{3}$ volume, this results in pressures of $\sim 1$ $\mathrm{TPa}$, but also in temperatures on the order of $\sim 10^{5} \mathrm{~K}(10 \mathrm{eV})$ with non-equilibrium heating-rates of $\sim 10^{17}-10^{18} \mathrm{~K} / \mathrm{s}(10 \mathrm{eV}$ in $100 \mathrm{fs}$ ) and fast cooling of $\sim 10^{14} \mathrm{~K} / \mathrm{s}$ (in $\sim 1 \mathrm{~ns}$ ). ${ }^{148,149}$ The processes during such a microexplosion are shown schematically in Fig. 6.

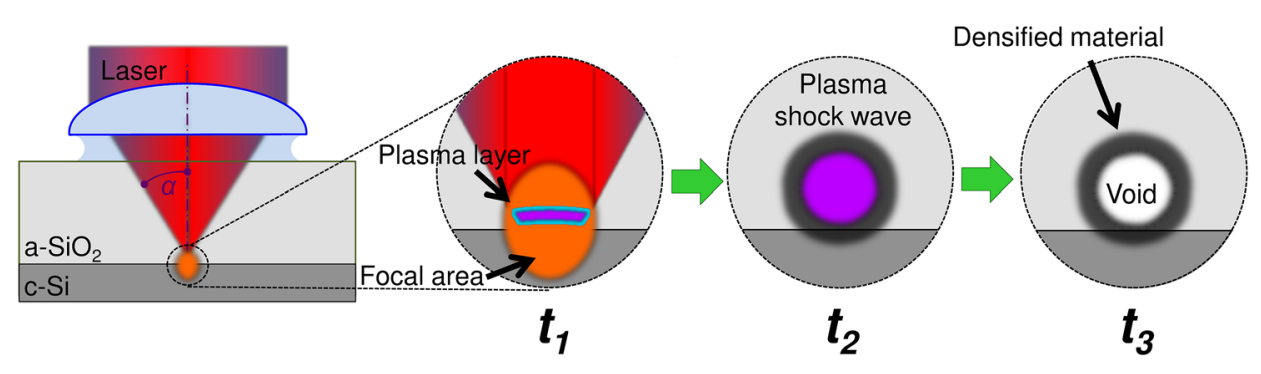

FIG. 6. A schematic of the processes during a laser-induced microexplosion within thermally grown amorphous silica on a crystalline silicon substrate based on the same processes induced in transparent materials. ${ }^{149}$ The key time frames are shown: the formation of the plasma layer in $\mathrm{t}_{1}$, the resulting shock wave in $t_{2}$, and the final void formation in $t_{3}$. See text for more details. 
During a single laser pulse, energy is deposited into the material, the material's atomic structure is destroyed, and localized plasma is induced within the $170 \mathrm{fs}$. For the particular case of $\mathrm{SiO}_{2}$ on $\mathrm{Si}$, it should thereby be noted that the energy is mainly absorbed into the $\mathrm{Si}$ and not the $\mathrm{SiO}_{2}$ due to the significantly lower ionization temperature of the former. After its formation, the localized plasma expands to result in the formation of a shock wave within $\sim 1-100$ ps. Thereby, the shock wave is stopped when the wave pressure becomes equal to the Young's modulus of the material ( $\sim 170 \mathrm{GPa}$ in the case of $\mathrm{Si}$ ). This is followed by a rarefunction wave accompanied by the formation of a void and densified material within the final stage of $\sim 100 \mathrm{ps}-1 \mathrm{~ns} .{ }^{148,149}$ After the full event, a laser-modified zone well beneath the surface of the material remains.

These laser-modified zones can be analyzed by electron microscopy techniques including scanning electron microscopy and transmission electron microscopy. While scanning electron microscopy can yield information about the size of voids created by different laser conditions, transmission electron microscopy yields structural information on the modifications that occurred within the Si. Due to the sample geometry with the region of interest $10 \mu \mathrm{m}$ beneath the surface, it is best to thin the sample to electron transparency using mechanical polishing to $\sim 70 \mu \mathrm{m}$ followed by focused ion beam processing akin to the previous indentation work. ${ }^{114}$ Transmission electron microscopy revealed the presence of phase-transformed volumes of Si beneath a void that formed in the $\mathrm{SiO}_{2}$. Electron diffraction analysis indicates a great number of reflection spots that cannot be matched to previously observed phases as shown in Fig. $7 .{ }^{17}$

The new phases were identified using the complimentary $a b$ initio random structure searches detailed in Sec. II A. These searches found the energetically competitive ST12 $\left(P 4_{1} 2_{1} 2, t P 12\right)$ and BT8 $\left(I 4_{1} / \mathrm{a}, t I 16\right)$ structures amongst the

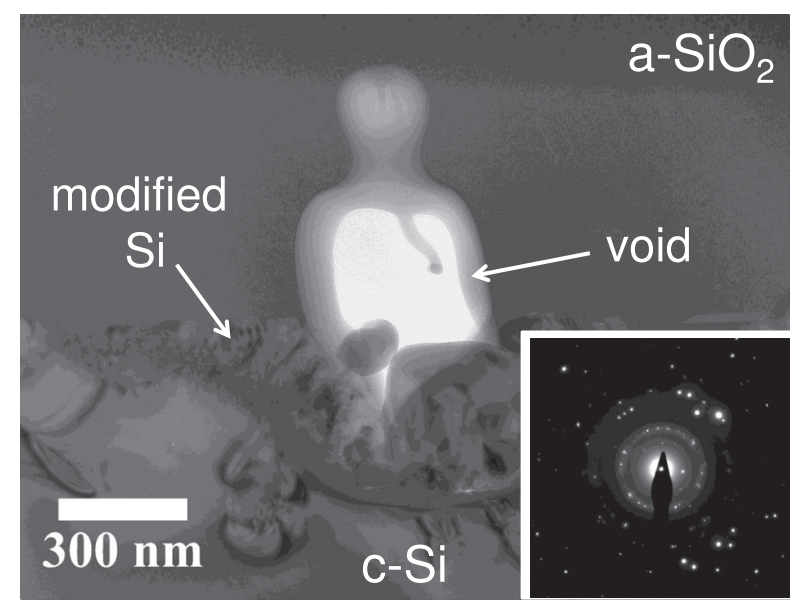

FIG. 7. Electron bright-field micrograph of the laser-modification in the $\mathrm{SiO}_{2} / \mathrm{Si}$ sample. A void is created in the top $\mathrm{SiO}_{2}$ layer, while modified material is visible in the Si substrate. An electron diffraction pattern of the modified zone in Si shown as inset reveals the presence of many diffraction reflections that cannot be attributed to DC-Si or known metastable phases of $\mathrm{Si}$ (see later for more detailed analysis of the diffraction pattern). The experimental details of the laser-induced modification are given in Ref. 17 and the figure is adapted with permission from Rapp et al., Nat. Commun. 6, 7555 (2015). Copyright 2015 Nature Publishing Group. known metastable phases and others. ${ }^{17}$ Thereby, the ST12 structure, a simple tetragonal structure with 12 atoms per unit cell, is well known to form in Si's sister element germanium upon pressure release. ${ }^{150}$ These two structures are shown in Fig. 8. The diffraction analysis performed by Rapp et al. revealed the formation of these two phases corresponding to an intriguing dependence on the laser fluence. While a higher fluence of $95 \mathrm{~J} / \mathrm{cm}^{2}$ yielded predominantly BT8-Si, a lower fluence of $48 \mathrm{~J} / \mathrm{cm}^{2}$ resulted in predominantly ST12-Si as evident from the diffraction patterns shown in Fig. 9. ${ }^{17}$

Although the work detailed above did not investigate the properties of these novel phases, ab initio calculations have indicated potentially highly useful functionality. Considerations of the phonon density of state and band structure characteristics obtained from DFT suggest that ST12 may possess an indirect bandgap between $1.1 \mathrm{eV}$ (Ref. 151) and $1.67 \mathrm{eV}$ (Ref. 34) and may exhibit superconductivity at, for $\mathrm{Si}$, relatively high temperatures if suitably doped. ${ }^{34} \mathrm{In}$ contrast, the BT8 structure may be a narrow bandgap semiconductor potentially useful for multiple exciton generation in photovoltaic applications akin to the prediction for $\mathrm{BC} 8$ $\mathrm{Si}$ and other experimentally known crystalline metastable phases. ${ }^{17}$

Interestingly, in the same laser modified zones, traces of two further tetragonal phases with 32 atoms in their unit cells may have been detected. ${ }^{17}$ As detailed earlier, one of these T32 structures with a space group of $P \overline{4} 2{ }_{1} c$ has been predicted to be suitable for solar applications due to a quasidirect band gap of close to $1.3 \mathrm{eV}^{3}$ Confirmation of the existence of this phase within the laser-modified volume would be clearly highly advantageous.

It is, however, not known yet whether phase-pure transformed volumes can be created with such laser-induced microexplosions although clearly their creation is the next crucial step for characterization of the functionality of these phases and thus to any future exploitation. Nonetheless, from these findings, it is abundantly clear that non-linear, nonequilibrium conditions very far from equilibrium in a confined environment enable the synthesis of novel functional structures of Si not previously observed.
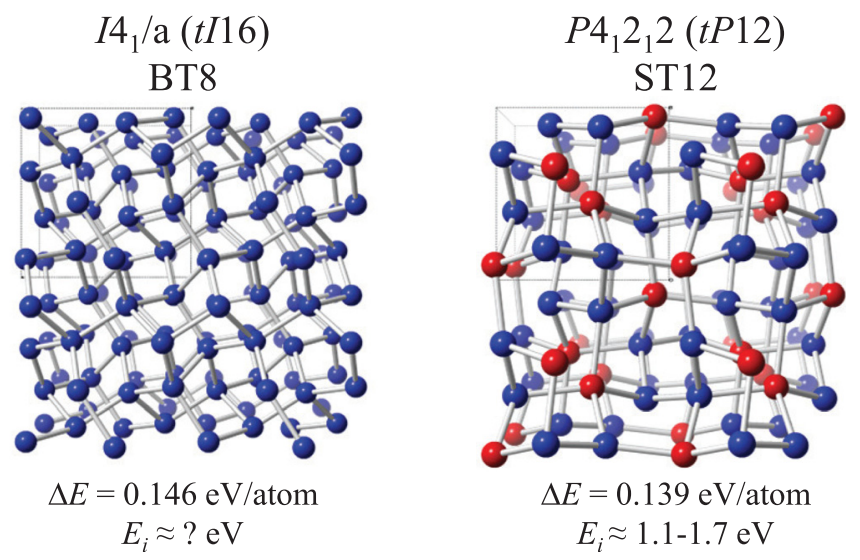

FIG. 8. Depiction of the crystal structures of BT8-Si and ST12-Si uncovered by ab initio random structure searches. ${ }^{17}$ Thereby, each image consists of four unit cells with lattice parameters of $\mathrm{a}=6.676 \AA$, $\mathrm{c}=6.522 \AA$ for BT8-Si and $\mathrm{a}=5.678 \AA, \mathrm{c}=6.825 \AA$ for ST12-Si. The figure is adapted from Ref. 17. 

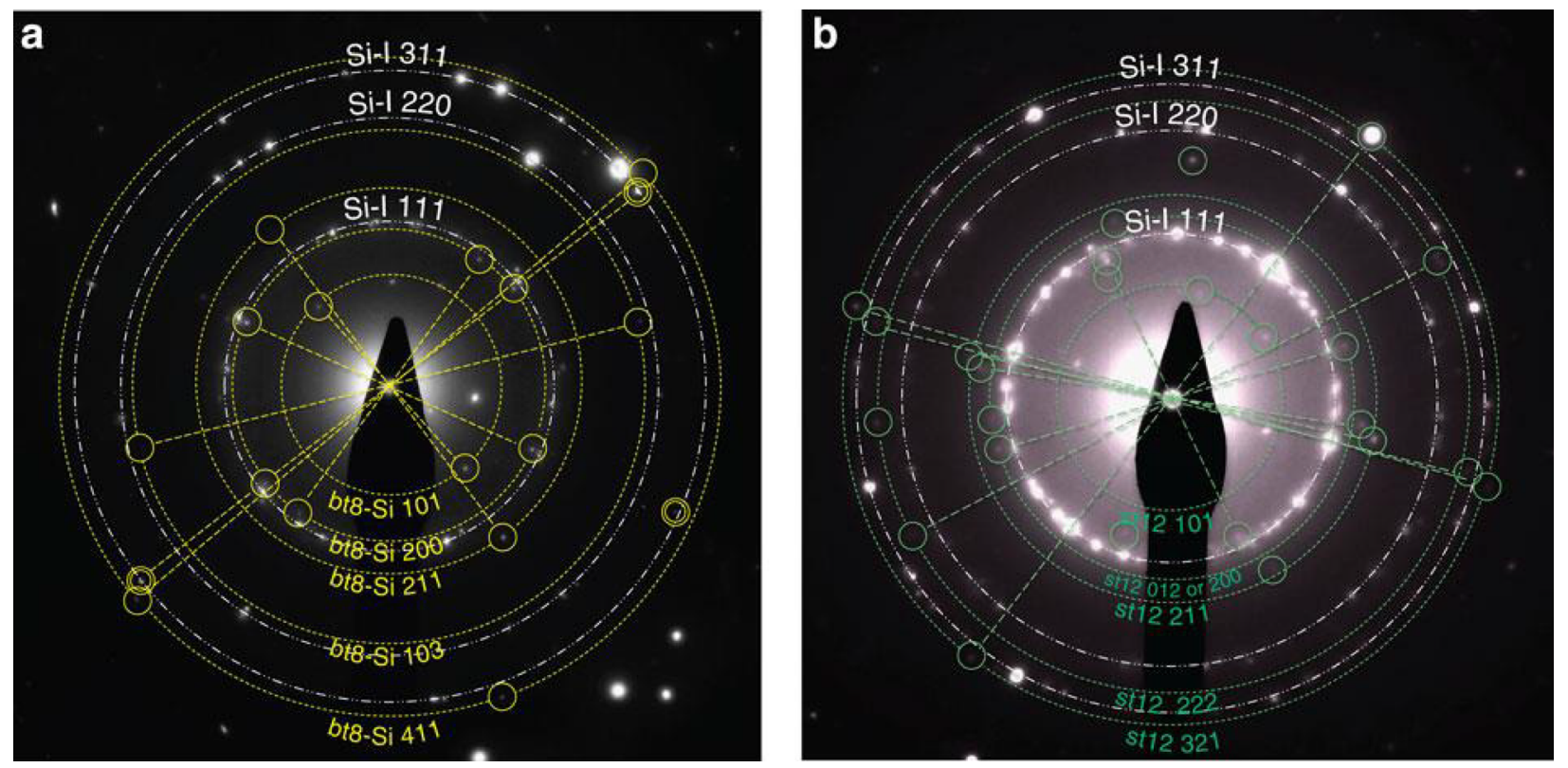

FIG. 9. Electron diffraction patterns taken from laser-modified zone created with (a) $95 \mathrm{~J} / \mathrm{cm}^{2}$ and (b) $48 \mathrm{~J} / \mathrm{cm}^{2}$ consisting predominantly of BT8-Si and ST12$\mathrm{Si}$, respectively. The diffraction rings of the metastable phases together with those of DC-Si are indicated with rings, while corresponding single crystal reflection pairs are connected with radial lines for clarity. Reprinted with permission from Rapp et al., Nat. Commun. 6, 7555 (2015). Copyright 2015 Nature Publishing Group.

\section{Synthesis from metastable precursors}

Chemical precursors enable convenient routes to access metastable states, as evidenced by much of organic chemistry. The general principle involves starting from an accessible metastable state (or one that becomes metastable through the synthetic process), thereby allowing access to broader regions of the energy landscape, normally inaccessible when starting from thermodynamic ground states.

Imagine a reaction coordinate starting from DC-Si (the thermodynamic ground state at ambient conditions) and ending at a metastable, exotic form. In this case, the change in free energy $\left(\Delta G_{f}\right)$ for all pathways will be positive. Some pathways might have small activation energy barriers $\left(E_{a}\right)$, e.g., small displacements of atoms from their equilibrium positions, and might be overcome by the application of stress, as was calculated for the case of BCT-5. ${ }^{62,152,153}$ Other pathways might have prohibitively large barriers, as would be expected for the cases with reconstructive transitions that require atomic diffusion over larger length scales (e.g., Ref. 81 for $(\beta-S n)-S i$ to DC-Si). Now imagine starting from a metastable precursor state. In this case, $\Delta G_{r x n}$ could be negative, and the reaction barrier might be smaller than the case of starting from the ground state. These pathways are depicted conceptually in Fig. 10.

These considerations have direct analogy to known transformations in bulk silicon. For example, upon decompression of metallic ( $\beta-\mathrm{Sn})-\mathrm{Si}$, the $\mathrm{R} 8$ and $\mathrm{BC} 8$ phases are observed rather than DC-Si. DC-Si remains the ground state structure, but $(\beta-\mathrm{Sn})$-Si can be thought of as a high-energy precursor phase with a smaller activation energy barrier to $\mathrm{R} 8 / \mathrm{BC} 8$ than DC-Si. Similarly, upon heating of BC8-Si at ambient conditions, HD-Si is formed before the appearance of DC-Si. Thus, the application of various pressure-temperature pathways alone is enough to access metastable forms of $\mathrm{Si}$ and can be qualitatively understood in terms of simple transition state kinetics.

Solid-state and aqueous-phase chemical reactions can also be understood within this context. Many silicon-based compounds and molecules possess different bonding topologies compared with DC-Si. A simple search of binary solidstate compounds containing silicon on the Inorganic Crystal Structure Database (ICSD) produces over 2400 results. In order to produce pure silicon allotropes from these compounds, their compositions must change, so chemical potential considerations must be taken into account along the reaction coordinate. Nevertheless, many compounds with different local structural motifs could provide low-energy pathways to novel silicon forms.

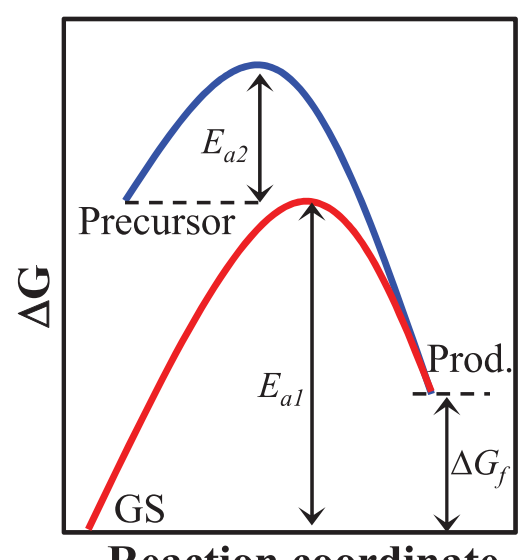

FIG. 10. Reaction pathways to a desired metastable product (Prod.) starting from the thermodynamic ground state (GS, red path) and from a metastable precursor (blue path). 


\section{Topochemical and colloidal methods}

Siloxene, while not purely silicon, is an example whereby precursor topology can be used to access a novel product. This structure, with composition $\mathrm{Si}_{6} \mathrm{H}_{3}(\mathrm{OH})_{3}$, is formed by etching $\mathrm{CaSi}_{2}$ in aqueous $\mathrm{HCl}^{154}$ This low-dimensional structure preserves the layered silicon motif found within $\mathrm{CaSi}_{2}$, but the absence of $\mathrm{Ca}^{2+}$ requires capping the ends with $\mathrm{H}$ and $\mathrm{OH}$. This material is reviewed in detail in another article in this issue $\mathrm{e}^{155}$ but readily shows how topochemistry can be used to preserve the Si bonding topologies found in precursor compounds.

Another example is so-called allo-Si, which, as far as we can tell, stands for "allotrope" Si. This phase was first reported in 1988 and is formed by the reaction of $\mathrm{Li}_{3} \mathrm{NaSi}_{6}$ with protic solvents. ${ }^{156}$ But first let us examine allo-Ge, a related material, because that story is clearer and the chemistry is similar.

Allo-Ge was first produced by Grüttner et al. in 1982 by the reaction of $\mathrm{Li}_{7} \mathrm{Ge}_{12}$ with benzophenone in tetrahydrofuran (THF) ${ }^{60}$ This work also produced a $4 H$-Ge structure by heating allo-Ge before it eventually transformed to Ge-I at higher temperature. $\mathrm{As} \mathrm{Li}$ is removed from the $\mathrm{Li}_{7} \mathrm{Ge}_{12}$ structure (see Fig. 11), topochemical relations between ${ }_{\infty}\left[\mathrm{Ge}_{12}\right]^{7-}$ units allow the formation of a novel Ge framework. A tentative structure for allo-Ge was proposed originally, but higherquality $\mathrm{Li}_{7} \mathrm{Ge}_{12}$ crystals were subsequently produced ${ }^{157}$ and the structure was determined with a larger degree of certainty. ${ }^{158,159}$ Allo-Ge (also called microcrystalline/m-allo-Ge) has extensive disorder along the $c$-axis associated with imperfect stacking and faults between layers. Nevertheless, diffraction patterns were indexed on an orthorhombic lattice with $a \approx 7.80 \AA, b \approx 16.27 \AA$, and $c \approx 11.91 \AA$, and experimental $\mathrm{XRD}$ and TEM data were explained by using the "GAa4" model proposed by Conesa, ${ }^{57}$ but with variation of $A-B$ stacking faults to account for the structural disorder. ${ }^{158,159}$

A similar situation applies to allo-Si, but the story is not as clear. Allo-Si was first produced by Von Schnering et al. in 1988 by the reaction of $\mathrm{Li}_{3} \mathrm{NaSi}_{6}$ with benzophenone in THF. ${ }^{156}$ This first report showed sharp diffraction lines and thermal stability up to $\sim 800 \mathrm{~K}$, but the precise structure was unresolved. In this case, ${ }_{\infty}^{2}\left[\mathrm{Si}_{6}\right]^{4-}$ units were thought to polymerize forming a three-dimensional silicon network. Fig. 11 shows the structural relationships by which these allotropes can be produced by etching metal from the precursor phases. A subsequent report by Zeilinger et al., however, suggested that this combination of silicon layers is not energetically favorable and amorphous/nanocrystalline products may be more likely than crystalline allo-Si. ${ }^{160}$ A well-defined and properly characterized crystalline form of allo-Si has yet to be produced, but several theoretical candidate structures are energetically feasible and topochemically sensible. ${ }^{57,160}$

From another end, novel forms of silicon may be produced through colloidal chemistry. In 2014, Ganguly et al. reported the synthesis of $\mathrm{BC} 8$ nanoparticles using a colloidal route. ${ }^{161}$ The process consisted of heating $\mathrm{SiI}_{4}$ in dioctylether, then reacting with $n$-butyllithium and 1-octanol at $280^{\circ} \mathrm{C}$ in an inert argon atmosphere. TEM images and powder XRD patterns of the resulting product revealed
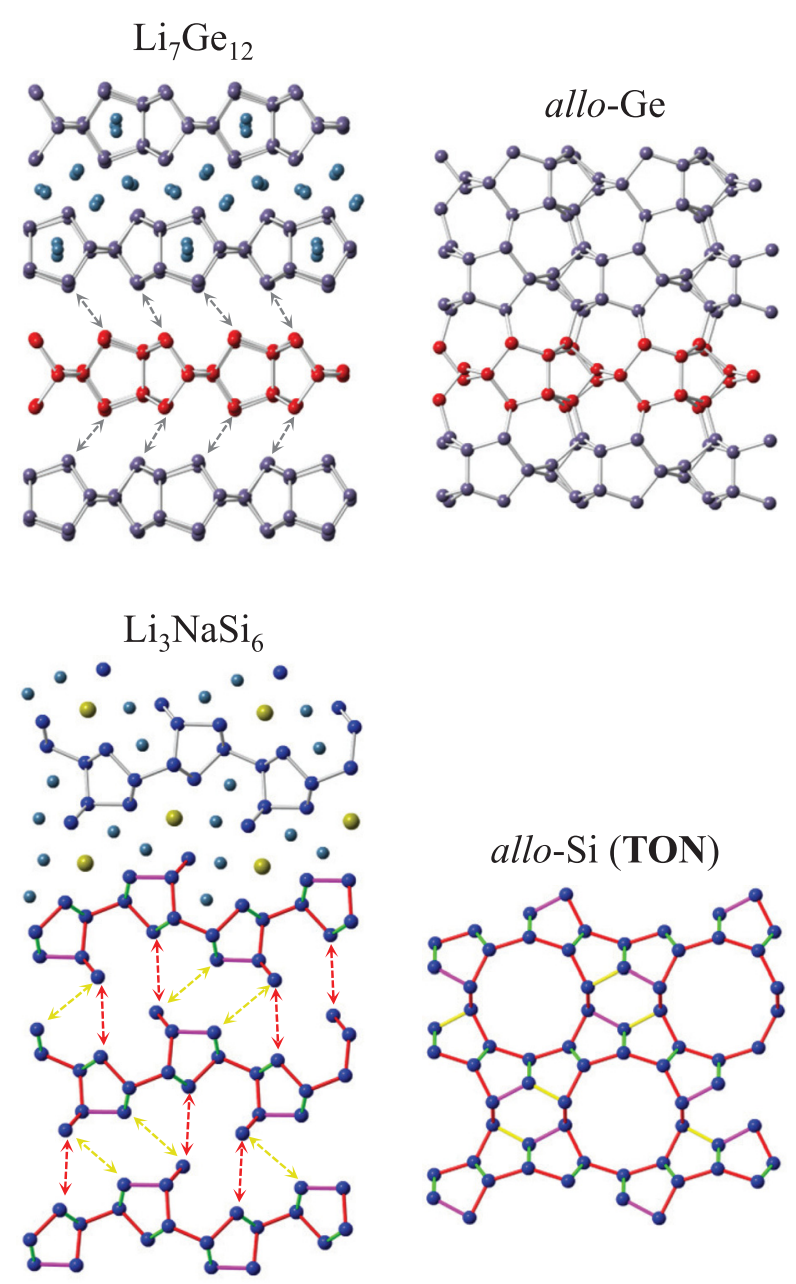

FIG. 11. Structures of the parents $\mathrm{Li}_{7} \mathrm{Ge}_{12}$ (Ref. 157) and $\mathrm{Li}_{3} \mathrm{NaSi}_{6}$ (Ref. 156) (on left) and the products allo-Ge ${ }^{158,159}$ and allo-Si TON ${ }^{57,160}$ (on right). The arrows and colored atoms/bonds help to guide the eye toward the topochemical relations between respective precursor and product. For the Ge case, the red spheres indicate examples of the same Ge atoms in the precursor before Li removal in the allo-Ge after Li removal. For the Si case, the colored arrows indicated in the $\mathrm{Li}_{3} \mathrm{NaSi}_{6}$ correspond to the newly formed bonds in allo-Si color-coded accordingly.

quasispherical particles of $\sim 5 \mathrm{~nm}$ with $d$-spacings that are consistent with the reported BC8 structure. ${ }^{162}$ The particles showed blue luminescence under UV light and exhibited visible absorption behavior consistent with a direct band gap near $2.8 \mathrm{eV}$. According to the theoretical calculations, $4 \mathrm{~nm}$ BC8 particles would exhibit a band gap of $1.37 \mathrm{eV}^{40}$ These differences could potentially be related to the particle "capping" materials: octanol in experiments and hydrogen in calculations. Nevertheless, colloidal synthesis routes might also serve as a valuable approach to access new forms of silicon. Indeed, this type of solution chemistry has been useful in the synthesis of exotic polymorphs in other systems (e.g., $\left.\mathrm{CuInS}_{2}\right) .{ }^{163}$

\section{Framework-based and high-pressure precursors}

Silicon clathrates are arguably the best known example of kinetically controlled synthesis of metastable silicon frameworks and have been discussed in detail previously, see, for example, Refs. 164-168. Originally discovered in 
1965 by Cros and coworkers ${ }^{50,169}$ via thermal decomposition of $\mathrm{Na}_{4} \mathrm{Si}_{4}$, the precise details of this transformation mechanism remain poorly understood. The Type-I (mep) and Type-II (mtn) phases, with ideal compositions of $\mathrm{Na}_{8} \mathrm{Si}_{46}$ and $\mathrm{Na}_{24} \mathrm{Si}_{136}$, are clearly metastable ones as only the $\mathrm{Na}_{4} \mathrm{Si}_{4}$ Zintl phase exists on the equilibrium $\mathrm{Na}-\mathrm{Si}$ binary phase diagram. ${ }^{170}$ DFT calculations reveal that $\mathrm{Na}_{4} \mathrm{Si}_{4}$ is a deep thermodynamic minimum, but the formation energies of the clathrate phases are small. For the Na-filled clathrates, thermodynamic stability is observed under high-pressure conditions (Fig. 12) ${ }^{171}$ Recently, multiple Na occupancy was observed for Type-II clathrates formed under high-pressure conditions. ${ }^{172}$

It is very interesting to note that despite the fact that Type-I and Type-II Na-Si clathrates are metastable phases, they are readily synthesized at ambient conditions. Because they are only truly thermodynamically stable under high pressure, this observation suggests that other high-pressure phases might be made at ambient conditions by starting from the appropriate chemical precursor. ${ }^{171}$

Hutchins et al. used in situ synchrotron X-ray diffraction to directly investigate the clathrate formation process. ${ }^{173}$ $\mathrm{Na}_{4} \mathrm{Si}_{4}$ was observed to transform directly to clathrate phases with no observable intermediates, suggesting a possible epitaxial relationship between precursor and product that facilitates the formation at low pressure. In some sense, the formation of filled clathrates is similar to the allo-Si $(\mathrm{Ge})$ phases described above. The precursor is a Zintl phase with negatively charged polyanionic silicon species, alkali metal

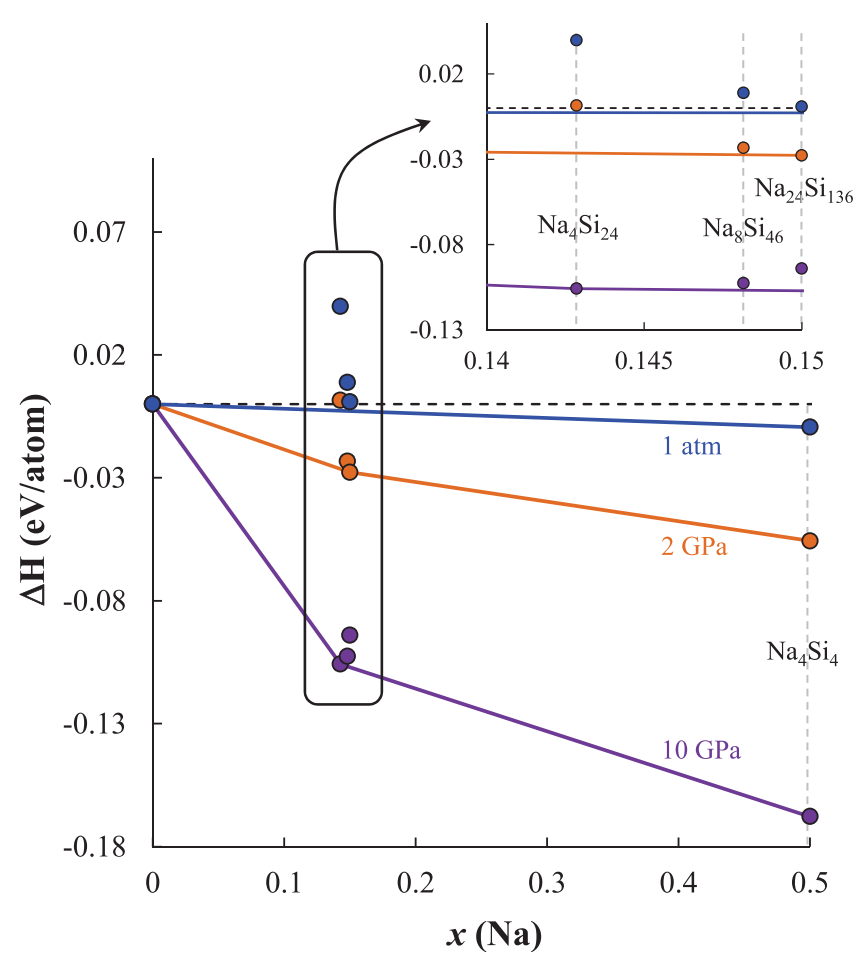

FIG. 12. Formation enthalpies of various Na-Si phases from DFT calculations $(0 \mathrm{~K}) . \mathrm{Na}_{4} \mathrm{Si}_{4}$ is a deep thermodynamic minimum, while three enthalpically competitive clathrate-type phases occupy a compositional range of $<1$ at. \% (inset). Clathrate phases are metastable at 1 atm but become favorable with increasing pressure. These calculations only allow single guest occupancy. This figure is created from data presented in Ref. 171. atoms are removed, and a new four-coordinated framework is formed. But in the case of clathrates, a significant portion of metal remains trapped inside of the polyhedral cages. In order to produce pure silicon allotropes from these materials, the metal must be removed.

Both physical and chemical methods have been used to remove metal atoms from the cages of silicon clathrates. By tuning the synthesis parameters, non-stoichiometric varieties are easily produced. ${ }^{50}$ The removal of additional metal typically involves heating in vacuum or chemical oxidation. ${ }^{174,175}$ It is notable that, while the Na content of Type-II clathrate can be reduced to ppm levels (and the framework can thus be considered a silicon allotrope) ${ }^{55} \mathrm{Na}$ has not successfully been removed from the cages of Type-I clathrate, which might be related to the sizes and interconnectivity of polyhedral cavities between the two structures.

Type II $\mathrm{Si}_{136}$ was successfully produced by Gryko et al. ${ }^{55}$ where $\mathrm{Na}_{x} \mathrm{Si}_{136}$ powders were subjected to several $\mathrm{HCl}$ washing/vacuum heating cycles and samples with $\mathrm{Na}$ content $<600 \mathrm{ppm}$ were isolated by centrifuging. Electrical conductivity and optical absorption measurements were used to demonstrate a wide band gap near $1.9 \mathrm{eV}$. Subsequently, Ammar et al. were able to reduce the Na content to $35 \mathrm{ppm}$ using a similar treatment including reaction with iodine. ${ }^{176}$

Another method to access novel metastable states is to use materials recovered from high pressure as precursors for subsequent manipulation. These high-pressure precursors (hpps) are formed under stability conditions at high pressure and represent high-energy starting points with different bonding patterns than compounds available at ambient conditions. Once the hpp is recovered to ambient pressure, it is treated in a separate physical/chemical process to create an entirely new material. New hpp starting points, which may easily be metastable from the ground state by $\sim 0.3 \mathrm{eV} /$ atom, allow for new reaction pathways to occur that would not be possible otherwise. This approach was recently used for the synthesis of " $\mathrm{Si}_{24}$ " (cas) and for the polymerization of methanide $\mathrm{C}^{4-}$ anions into longer $\mathrm{C}_{3}{ }^{4-}$ allylenide chains. ${ }^{16,177}$ Another analogous example is the heating of $\mathrm{BC} 8-\mathrm{Si}$, which is recovered from high-pressure, to produce HD-Si (lon) at ambient pressure. ${ }^{30}$

For the case of $\mathrm{Si}_{24}$ (cas), the precursor phase, $\mathrm{Na}_{4} \mathrm{Si}_{24}$, was first produced at high pressure $\left(\sim 8 \mathrm{GPa}\right.$ and $\left.850^{\circ} \mathrm{C}\right)$. The cas lattice contains open channels along the $a$-axis, produced by eight- and six-membered silicon rings, which help to facilitate sodium diffusion. Once recovered to ambient pressure, $\mathrm{Na}$ transport through the channels was observed at temperatures as low as $40^{\circ} \mathrm{C}$, while complete $(<1000 \mathrm{ppm}) \mathrm{Na}$ removal was observed after vacuum degassing at $125^{\circ} \mathrm{C}$ at $10^{-5}$ Torr for eight days. ${ }^{16}$ This is in contrast with Na removal in type-II clathrate, which requires temperatures $>400^{\circ} \mathrm{C}$; the open channels in cas likely provide a much smaller barrier for Na diffusion compared with the "closed" polyhedral clathrate cavities. Fig. 13 depicts the open-framework $\mathrm{Na}_{4} \mathrm{Si}_{24}$ structure and conceptual picture of diffusion to produce $\mathrm{Si}_{24}$.

Similar to the case of stoichiometric Type-I and Type-II $\mathrm{Na}-\mathrm{Si}$ clathrates, fully occupied $\mathrm{Na}_{4} \mathrm{Si}_{24}$ is metallic but undergoes a metal-to-insulator transition upon sodium removal. In the semiconducting state, $\mathrm{Si}_{24}$ possess a 


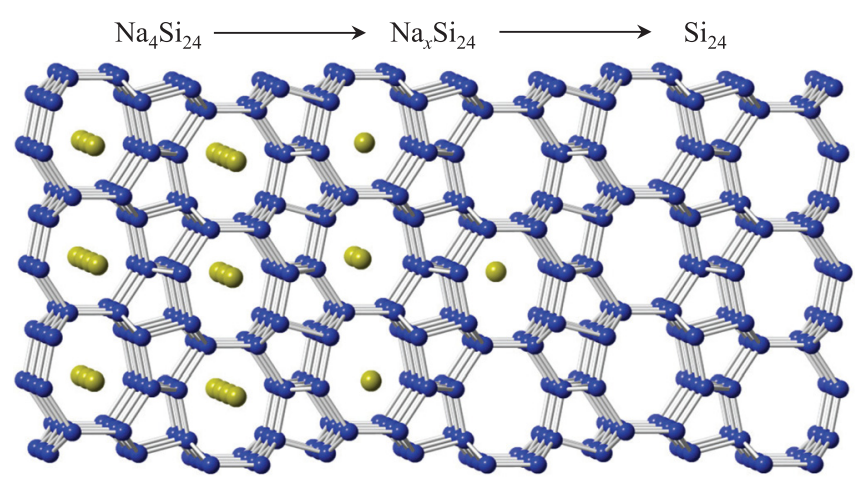

FIG. 13. $\mathrm{Na}_{4} \mathrm{Si}_{24}$ structure with open-framework silicon lattice showing $\mathrm{Na}$ diffusion pathway to produce $\mathrm{Si}_{24}{ }^{16}$

quasidirect bandgap near $1.4 \mathrm{eV} \quad\left(E_{i}=1.30 \mathrm{eV}\right.$ and $E_{d}=1.34 \mathrm{eV}$ from calculations under the $\mathrm{GW}$ approximation, Fig. 14). Notably, $1.34 \mathrm{eV}$ is the optimal value for solar conversion efficiency under the Shockley-Queisser limit ${ }^{178}$ and $\mathrm{Si}_{24}$ exhibits visible light absorption behavior comparable to materials that are regarded as excellent thin film absorbers. ${ }^{16}$ If the synthesis of this material can be optimized, it could open the possibility for silicon-based thinfilm solar absorbers that maintain the intrinsic advantages of earth-abundant $\mathrm{Si}$ but operate at thicknesses reduced by two orders of magnitude.

\section{PERSPECTIVES FOR THE FIELD}

As the controlled synthesis of new materials with tailored functionality is vital to meet future technological and societal needs, there is a strong justification to look beyond the standard methods of synthesis. Silicon is a material ripe for exploitation for phase-engineering to create high-efficiency materials for a range of targeted applications including solar power conversion. The keen interest is underpinned by its high abundance, non-toxicity, and its extremely wide-spread use in semiconductor and solar cell technology and the resulting multi-billion dollar infrastructure in $\mathrm{Si}$ based processing plants already in existence. The application of pressure represents a powerful tool for the synthesis of
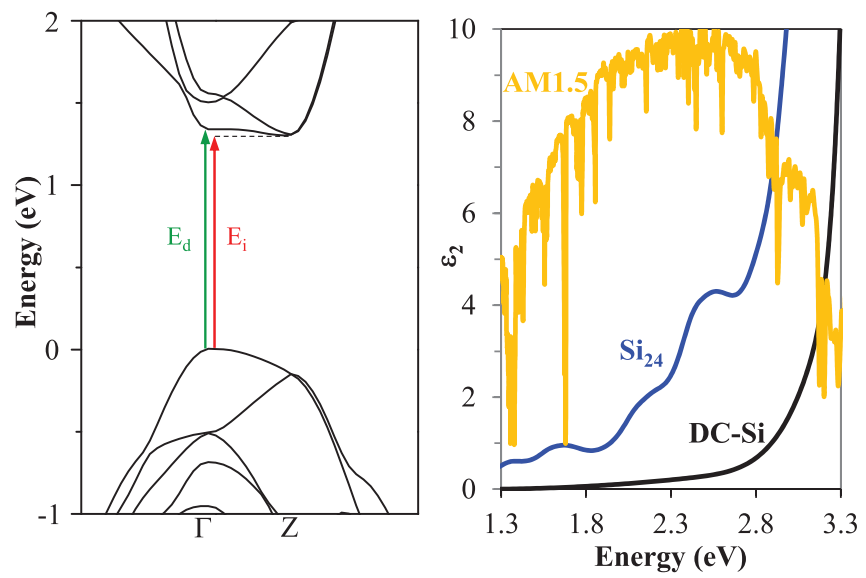

FIG. 14. Portion of the calculated $\mathrm{Si}_{24}$ electronic band structure (GW) showing the quasidirect nature of the band gap (left) and imaginary portion of the dielectric function compared with the AM1.5 solar spectrum (right). The figure shown here is based on the data presented in Ref. 16. novel Si materials with tailored physical properties and functionality. This is achieved by enabling access to local minima on the $\mathrm{Si}$ free-energy landscape with potential for metastable recovery of useful new Si allotropes.

As detailed above, standard static pressure conditions in a DAC yield the synthesis of BC8- (to some extent), R8-, and HD-Si from a standard DC-Si (or a-Si) precursor. ${ }^{30,37}$ $A b$ initio calculations predict great potential for photovoltaic or other optoelectronic applications for these three polymorphs under optimized conditions. ${ }^{33,40,41}$ Furthermore, a plethora of new metastable, functional allotropes has been predicted through various $a b$ initio methods (see Section IB). Until recently, however, the synthesis of further useful allotropes has been hampered by strong kinetic barriers and no new metastable crystalline structures were identified in over $20 \mathrm{yr}$. This limit has been overcome through the use of alternative methods for pressure application and the utilization of novel precursor-based pathways with inherent metastability.

Point loading has yielded a significantly increased portion of R8-Si to-be-utilized outside of the pressure applicator. ${ }^{39}$ The unique stress conditions combined with the absence of confinement on such phase transformed volumes created by point loading have enabled the synthesis of another polymorph, Si-XIII. ${ }^{130}$ This polymorph may well be the first low-density phase of Si (i.e., density lower than DC$\mathrm{Si}$ ) that has been formed via a (de-)compression-annealing pathway from DC-Si. Furthermore, laser-induced microexplosions that apply non-equilibrium conditions in a highly confined environment have yielded at least two more 4-fold coordinated polymorphs, ST12-Si and BT8-Si. ${ }^{17}$ There are now numerous predictions for additional structures with close to ideal direct/quasidirect band gaps and feasible stabilities, and the application of high pressure to chemical highpressure precursors has resulted in one of them: $\mathrm{Si}_{24}$, with a quasidirect band gap close to $1.4 \mathrm{eV} .^{16}$

These exciting observations of new functional allotropes of $\mathrm{Si}$ beg a number of questions. Can further pathways be facilitated based on these findings? Can the synthesis of these allotropes be "scaled-up" sufficiently to be of technological use? Can these synthesis pathways be transferred to other materials? These questions which represent key challenges but also opportunities for the field are discussed now in some detail.

\section{A. Accessing additional functional allotropes}

The new methods for syntheses of functional Si allotropes described above raise the question whether additional allotropes could be created using similar methods. Clearly, laser-induced microexplosions with the tantalizing possibility of the synthesis of a T32 structure with a quasidirect bandgap of $1.3 \mathrm{eV}$ promise access to further allotropes. The finding presented in Ref. 17 suggests an energy dependence resulting in correlating densities: The BT8 phase resulting from a higher laser energy has a higher density (17\% more dense than DC-Si) and the ST12 phase resulting from a lower energy also possesses a higher density, but reduced from BT8-Si (6\% more dense than DC-Si). Hence, by 
increasing the density of the parent $\mathrm{Si}$ phases (simply through increasing the energy of the laser pulse and thus the pressure), it seems likely that further polymorphs can be quenched. However, to achieve control over the phase formation, it will be critical to fully understand the laser-sample interaction during such microexplosions.

Another method to access different allotropes would be the use of further metastable precursors. One possibility could be the use of amorphous precursors, i.e., amorphous silicon. Amorphous $\mathrm{Si}$ is inherently metastable and sufficiently versatile to be designed into a variety of appropriately tuned precursors. ${ }^{128,179}$ Furthermore, a-Si is readily prepared in a hydrogenated form ${ }^{179}$ thus opening the realm for the synthesis of hydrogen-rich $\mathrm{Si}$ structures which may be highly advantageous for solar applications. ${ }^{40}$

Sodium-filled silicon clathrates, while only thermodynamically stable at high pressure, are readily synthesized on the gram scale at low pressure. ${ }^{180}$ This clearly suggests promise for new precursor phases that could allow access to more exotic forms. Sodium removal from these structures does represent one major challenge from the perspective of device-grade semiconductors for microelectronic or optoelectronic applications (see, for example, the recent review by Krishna and $\mathrm{Koh}^{181}$ ). Empty germanium clathrates, on the other hand, have been produced by chemical methods without the need for subsequent guest atom removal. ${ }^{182}$ $\mathrm{Na}_{4} \mathrm{Ge}_{9}$ was reacted at $300{ }^{\circ} \mathrm{C}$ in a eutectic mixture of dodecyltrimethylammonium chloride and aluminiumtrichloride in a 1:1 molar ratio, resulting in the oxidation $\mathrm{Ge}_{9}{ }^{4-}$ anions and the direct formation of guest-free type-II Ge clathrates. Optical absorption measurements revealed $\mathrm{Ge}_{136}$ to possess a direct band gap of $0.6 \mathrm{eV}$. This observation suggests that similar routes could be designed for the case of silicon where the need for subsequent $\mathrm{Na}$ removal would be eliminated.

The area of high-pressure precursors is almost entirely unexplored and more routes will inevitably be discovered. There is great potential for novel silicon-based binary compounds formed under high-pressure conditions that could serve as metastable precursor to access new silicon framework topologies. Similarly, there is a broad space available to optimize the synthesis of exotic core structure silicon nanoparticles since the initial report of $\mathrm{BC} 8-\mathrm{Si}^{161}$

Finally, in the current review, pressure was considered as the predominant variable for the access to metastable states far form equilibrium. Chemical reactivity (and hence synthesis capabilities) is greatly enhanced if heating is added. Indeed, many new materials have been identified via combined high-temperature, high pressure application such as diamond and cubic boron nitride, ${ }^{183-185}$ but also solid nitrogen, ${ }^{186}$ new cubic forms of Group 14 nitrides, ${ }^{187-189}$ and metal and boron-carbon nitrides. ${ }^{190}$ The potential of additional high-temperature conditions has been poorly explored for the synthesis of metastable $\mathrm{Si}$ allotropes thus opening a new avenue for investigation.

\section{B. Scale-up to technologically interesting levels}

The amount of material synthesized in a standard DAC is extremely small, for Si only in the order of $0.01 \mathrm{mg} .^{20,21}$
This could be relatively easily scaled up to $\sim 1 \mathrm{mg}$ per pressure run with (ultra-) large-volume DACs as used and further developed for neutron diffraction. ${ }^{126,191}$ If pressures below $\sim 20 \mathrm{GPa}$ are sufficient (as is the case for most of the present day metastable Si allotropes), the larger volume presses such as a Paris-Edinburg cell ${ }^{192}$ or a multi-anvil press ${ }^{193}$ could be utilized instead for $\mathrm{Si}$ amounts on the order of $100 \mathrm{mg}$. Furthermore, large-volume presses such as "belts" or "bars" have been highly successful on an industrial level for the synthesis of diamond or cubic boron nitride at $\sim 5 \mathrm{GPa} .{ }^{194,195}$ The initial synthesis of these materials also occurred at $10-20 \mathrm{GPa}^{183-185}$ but was lowered significantly through the use of suitable catalysts. Thus, identification of similar catalysts for $\mathrm{Si}$ or use of optimized precursor materials could also yield synthesis pressures in the technologically interesting regime of up to $\sim 5 \mathrm{GPa}$.

Another way to optimize the synthesis yield is not the simple increase of sample volume, but the creation of phase transformed volumes in useful sizes. In the current climate of miniaturization of semiconductor devices, it is not necessarily needful to create one large pellet of a phase transformed volume as made in a press. Instead, it can be equally fruitful to create phase transformed volumes in suitably designed patterns within a Si wafer, for example. As indicated above, indentation and laser-induced microexplosions result in phase transformed volumes directly created in standard Si wafers with technologies compatible with modern semiconductor fabrication facilities already used in semiconductor industry. Overlapping of indentations can be used for electronic nanopatterning via the formation of conductive (or insulating) lines. ${ }^{39,196}$ If instead of a single indenter an "indenter stamp" would be used, such a process could be easily scaled-up to technologically feasible sizes. Similarly, laser-modified volumes could be overlapped to create similar nanopatterning in the $\mathrm{Si}$ substrate. Especially, if higher throughput lasers are used (as in standard industrial laser processing), ${ }^{197}$ such process would be equally scalable to industrial levels.

Deposition may also prove to be a useful frontier for exotic silicon phases and enable scalability to useful levels. As mentioned above, chemical vapor deposition has proved valuable for carbon diamond growth, another phase that is only thermodynamically stable at high pressure, but now produced below atmospheric pressure in bulk, ${ }^{198,199}$ and similar techniques might be employed for silicon. Hauge et al. recently used epitaxial relations with $\mathrm{GaP}$ and the "crystal transfer" method to grow HD-Si core-shell nanowires. ${ }^{200}$ This finding suggests great promise for growing other types of exotic silicon on appropriate substrates. Deposition methods could also eliminate the need for post processing (e.g., $\mathrm{Na}$ removal from clathrates) that results in degenerate semiconductor behavior.

Thus, it seems feasible that an engineering solution will be found to tackle the industrially useful synthesis of a metastable Si allotrope provided that one of sufficient technological potential is identified. Indeed, syntheses of diamond and cubic boron nitride provide the prime examples for such a procedure. Initial synthesis pressures and temperatures were beyond industry, but keen interest in these two superhard 
materials drove the modern possibility of their low-pressure growth via chemical vapor deposition, entirely without the need for pressure. ${ }^{198,199,201}$

\section{Transferability to other materials}

Silicon's sister elements on the periodic table, carbon and germanium, possess similarly rich energy landscapes with many (local) minima accessible by extreme conditions. In the case of carbon, only the formation of the perfect tetrahedrally bonded polymorphs diamond and lonsdaleite has been achieved from the lowest-energy equilibrium graphite. $^{183,184,202}$ Access to minima beyond these two polymorphs requires ultra-high pressures that are not readily accessible under static compression of crystalline precursors making evidence of the predicted BC8- or R8-carbon ${ }^{6,203}$ scarce. This limitation could be overcome through suitable non-equilibrium shocks or through the use of optimized chemical high-pressure precursors.

In contrast to $\mathrm{C}$, the energy landscape of $\mathrm{Ge}$ is more readily accessible. Indeed, the same standard metastable polymorphs (ST12-Ge, BC8-Ge, R8-Ge, and HD-Ge) have been formed through static (de-)compression in a DAC. ${ }^{204-206}$ Interestingly, indentation has proven particularly useful for the synthesis of R8-Ge from an amorphous Ge precursor and was in fact able to fully proof its existence of the first time. ${ }^{207}$ Furthermore, Ge is analogous to $\mathrm{Si}$ in its metallization pathways and hence formation of further metastable polymorphs may be expected through laser-induced microexplosions. ${ }^{13,14,23}$ Equally, similar chemical precursor methods are directly transferrable between $\mathrm{Si}$ and $\mathrm{Ge}$, as highlighted above. Consequently, we expect that a number of low-energy Ge structures will be realized experimentally through similar precursor-based pathway methods.

Finally, these methods are not limited to Group 14 elements. All compound semiconductors, i.e., the binary equivalents of Group 14 elements, possess (local) minima in their energy landscapes and exhibit phase transitions under pressure. ${ }^{13,14}$ Thus, most likely, the methods presented here can also yield further metastable allotropes in this large family of technically important materials.

\section{CLOSING REMARKS}

This review has highlighted in some depth the rich complexity of the free-energy landscape of the Group 14 element silicon and the many opportunities this represents for the synthesis of new, exotic metastable Si allotropes with exciting functionality. As detailed here, an impressive multitude of functional $\mathrm{Si}$ allotropes has been predicted over the last years, allotropes very similar in energetics to experimentally known phases and thus likely metastable once recovered to ambient conditions, or readily synthesizable through other clever experimental pathways. However, structure prediction cannot easily shed light on how to synthesize these allotropes experimentally and thus the key challenge for many years has been experimentally accessing these local minima. This current review has detailed three innovative approaches to synthesis that overcome strong kinetic barriers and enable recovery of novel exotic structures of $\mathrm{Si}$. These new approaches have yielded three structurally characterized new allotropes, at least one with useful functionality for solar power conversion with some evidence of further, not fully identified, allotropes in existence.

In addition for reviewing these previous findings, this current article has also given thoughts on the future perspectives for high-pressure synthesis and beyond. Excitingly, the limitations of the novel approaches reviewed here are entirely unknown. Since realization of their full potential promises access to further functional Si allotropes, several avenues were suggested. A key challenge of high-pressure synthesis is always the question of scalability to industrial levels. Progress has been made in this regard over the last years and further possibilities were outlined. It seems apparent that engineering solutions to the scalability problem can be found once a sufficiently powerful driver, e.g., uniquely useful $\mathrm{Si}$ allotropes, is created. Furthermore, the methods reviewed here are in no way limited to exotic $\mathrm{Si}$ allotropes and hence promising outlooks for other material systems such as carbon and germanium but also compound semiconductors have been provided.

Thus, it is clear that the synthesis of exotic Si allotropes using these powerful variants of synthesis through extreme conditions represents the beginnings of a wide-open field with exciting prospects yet to be realized.

\section{ACKNOWLEDGMENTS}

We thank M. Amsler (Northwestern University, USA) for assistance with Figure 3.

B.H. gratefully acknowledges full funding from an Alvin M. Weinberg Fellowship (ORNL) and the Spallation Neutron Source (ORNL), sponsored by the U.S. Department of Energy, Office of Basic Energy Sciences. ORNL is funded under DOE-BES Contract No. DE-AC05-00OR22725; the SNS is supported by the Scientific User Facilities division, DOE-BES under Contract No. DE-AC05-00OR22725 and the Alvin M. Weinberg Fellowship by the ORNL LDRD scheme under Project No. 7620.

T.A.S. acknowledges support from Energy Frontier Research in Extreme Environments (EFree) Center, an Energy Frontier Research Center funded by the U.S. Department of Energy, Office of Science under Award No. DE-SC0001057.

J.E.B. would like to acknowledge the Australian Research Council for a Future Fellowship and funding under the Discovery Project scheme.

${ }^{1}$ S. Botti, J. A. Flores-Livas, M. Amsler, S. Goedecker, and M. A. L. Marques, Phys. Rev. B 86, 121204 (2012).

${ }^{2}$ A. Mujica, C. J. Pickard, and R. J. Needs, Phys. Rev. B 91, 214104 (2015).

${ }^{3}$ Q. Zhu, A. R. Oganov, A. O. Lyakhov, and X. X. Yu, Phys. Rev. B 92 , 024106 (2015).

${ }^{4}$ M. Amsler, S. Botti, M. A. L. Marques, T. J. Lenosky, and S. Goedecker, Phys. Rev. B 92, 014101 (2015).

${ }^{5}$ N. W. Ashcroft, Phys. Rev. Lett. 21, 1748 (1968).

${ }^{6}$ M. T. Yin, Phys. Rev. B 30, 1773-1776 (1984).

${ }^{7}$ A. Y. Liu and M. L. Cohen, Science 245, 841 (1989).

${ }^{8}$ R. N. Noyce, "Semiconductor device and lead structure," US Patent No. US2981877 A (1961).

${ }^{9}$ J. S. Kilby, “Miniaturized electronic circuits,” US Patent No. US3138743 A (1964) 
${ }^{10}$ D. E. Carlson and C. R. Wronski, Appl. Phys. Lett. 28, 671-374 (1976).

${ }^{11}$ M. A. Green, Solar Cells: Operating Principles, Technology, and System Applications (Prentice-Hall, Inc., Englewood Cliffs, NJ, 1983).

${ }^{12}$ M. A. Green, K. Emery, Y. Hishikawa, W. Warta, and E. D. Dunlop, Prog. Photovoltaics 23, 1 (2015).

${ }^{13}$ G. J. Ackland, Rep. Prog. Phys. 64, 483-516 (2001).

${ }^{14}$ A. Mujica, A. Rubio, A. Munoz, and R. J. Needs, Rev. Mod. Phys. 75, 863-912 (2003)

${ }^{15}$ H. Katzke and P. Tolédano, J. Phys.: Condens. Matter 19, 275204 (2007).

${ }^{16}$ D. Y. Kim, S. Stefanoski, O. O. Kurakevych, and T. A. Strobel, Nat. Mater. 14, 169-173 (2015).

${ }^{17}$ L. Rapp, B. Haberl, C. J. Pickard, J. E. Bradby, E. G. Gamaly, J. S. Williams, and A. V. Rode, Nat. Commun. 6, 7555 (2015).

${ }^{18}$ M. O'Keeffe, M. A. Peskov, S. J. Ramsden, and O. M. Yaghi, Acc. Chem. Res. 41, 1782-1789 (2008).

${ }^{19}$ P. Debye and P. Scherrer, Phys. Z. 17, 277-283 (1916).

${ }^{20}$ R. Boehler, Mater. Today 8, 34-42 (2005).

${ }^{21}$ A. Jayaraman, Rev. Mod. Phys. 55, 65 (1983).

${ }^{22}$ J. S. Smith, S. V. Sinogeikin, C. Lin, E. Rod, L. Bai, and G. Shen, Rev. Sci. Instrum. 86, 072208 (2015).

${ }^{23}$ J. C. Jamieson, Science 139, $762-764$ (1963).

${ }^{24}$ M. I. McMahon and R. J. Nelmes, Phys. Rev. B 47, 8337-8340 (1993).

${ }^{25}$ M. I. McMahon, R. J. Nelmes, N. G. Wright, and D. R. Allan, Phys. Rev. B 50, 739-743 (1994).

${ }^{26}$ H. Olijnyk, S. K. Sikka, and W. B. Holzapfel, Phys. Lett. A 103, 137-140 (1984).

${ }^{27}$ M. Hanfland, U. Schwarz, K. Syassen, and K. Takemura, Phys. Rev. Lett. 82, 1197-1200 (1999)

${ }^{28}$ S. J. Duclos, Y. K. Vohra, and A. L. Ruoff, Phys. Rev. B 41, 12021-12028 (1990).

${ }^{29}$ S. Minomura and H. G. Drickamer, J. Phys. Chem. Solids 23, 451-456 (1962)

${ }^{30}$ R. H. Wentorf and J. S. Kasper, Science 139, 338 (1963).

${ }^{31}$ J. M. Besson, E. H. Mokhtari, J. Gonzalez, and G. Weill, Phys. Rev. Lett. 59, 473-476 (1987).

${ }^{32}$ J. D. Joannopoulos and M. L. Cohen, Phys. Lett. A 41, 71-72 (1972).

${ }^{33}$ B. D. Malone, J. D. Sau, and M. L. Cohen, Phys. Rev. B 78, 161202 (2008).

${ }^{34}$ B. D. Malone, J. D. Sau, and M. L. Cohen, Phys. Rev. B 78, 035210 (2008).

${ }^{35}$ G. Weill, J. L. Mansot, G. Sagon, C. Carlone, and J. M. Besson, Semicond. Sci. Technol. 4, 280-282 (1989).

${ }^{36}$ Y. X. Zhao, F. Buehler, J. R. Sites, and I. L. Spain, Solid State Commun. 59, 679-682 (1986).

${ }^{37}$ J. Crain, G. J. Ackland, J. R. Maclean, R. O. Piltz, P. D. Hatton, and G. S. Pawley, Phys. Rev. B 50, 13043-13046 (1994).

${ }^{38}$ R. O. Piltz, J. R. Maclean, S. J. Clark, G. J. Ackland, P. D. Hatton, and J. Crain, Phys. Rev. B 52, 4072-4085 (1995).

${ }^{39}$ S. Ruffell, K. Sears, A. P. Knights, J. E. Bradby, and J. S. Williams, Phys. Rev. B 83, 075316 (2011).

${ }^{40}$ S. Wippermann, M. Vörös, D. Rocca, A. Gali, G. Zimanyi, and G. Galli, Phys. Rev. Lett. 110, 046804 (2013).

${ }^{41}$ C. Rödl, T. Sander, F. Bechstedt, J. Vidal, P. Olsson, S. Laribi, and J. F. Guillemoles, Phys. Rev. B 92, 045207 (2015).

${ }^{42}$ M. Wilson and P. F. McMillan, Phys. Rev. Lett. 90, 135703 (2003).

${ }^{43}$ D. Daisenberger, P. F. McMillan, and M. Wilson, Phys. Rev. B 82, 214101 (2010)

${ }^{44}$ M. Beekman, Mater. Today 18, 304-305 (2015).

${ }^{45}$ A. F. Wells, Three-Dimensional Nets and Polyhedra (Wiley, New York, 1977).

${ }^{46}$ O. D. Friedrichs, A. W. M. Dress, D. H. Huson, J. Klinowski, and A. L. Mackay, Nature 400, 644-647 (1999).

${ }^{47}$ O. Delgado-Friedrichs, M. D. Foster, M. O'Keeffe, D. M. Proserpio, M. M. J. Treacy, and O. M. Yaghi, J. Solid State Chem. 178, 2533-2554 (2005)

${ }^{48}$ M. A. Zwijnenburg, K. E. Jelfs, and S. T. Bromley, Phys. Chem. Chem. Phys. 12, 8505-8512 (2010).

${ }^{49}$ R. Nesper, K. Vogel, and P. E. Blochl, Angew. Chem., Int. Ed. Engl. 32, 701-703 (1993).

${ }^{50}$ J. S. Kasper, P. Hagenmul, M. Pouchard, and C. Cros, Science 150, 1713 (1965)

${ }^{51}$ G. B. Adams, M. O'Keeffe, A. A. Demkov, O. F. Sankey, and Y.-M. Huang, Phys. Rev. B 49, 8048 (1994).
${ }^{52}$ A. A. Demkov, W. Windl, and O. F. Sankey, Phys. Rev. B 53, 11288 (1996).

${ }^{53}$ K. Moriguchi, S. Munetoh, and A. Shintani, Phys. Rev. B 62, 7138 (2000).

${ }^{54}$ X. Blase, Phys. Rev. B 67, 035211 (2003).

${ }^{55}$ J. Gryko, P. F. McMillan, R. F. Marzke, G. K. Ramachandran, D. Patton, S. K. Deb, and O. F. Sankey, Phys. Rev. B 62, R7707-R7710 (2000).

${ }^{56}$ D. Connetable, Phys. Rev. B 82, 075209 (2010).

${ }^{57}$ J. C. Conesa, J. Phys. Chem. B 106, 3402-3409 (2002).

${ }^{58}$ A. J. Karttunen, T. F. Fassler, M. Linnolahti, and T. A. Pakkanen, Inorg. Chem. 50, 1733-1742 (2011).

${ }^{59}$ D. J. Chadi, Phys. Rev. B 32, 6485-6489 (1985).

${ }^{60}$ A. Gruttner, R. Nesper, and H. G. Vonschnering, Angew. Chem., Int. Ed. Engl. 21, 912-913 (1982).

${ }^{61}$ I. A. Baburin, D. M. Proserpio, V. A. Saleev, and A. V. Shipilova, Phys. Chem. Chem. Phys. 17, 1332-1338 (2015).

${ }^{62}$ L. L. Boyer, E. Kaxiras, J. L. Feldman, J. Q. Broughton, and M. J. Mehl, Phys. Rev. Lett. 67, 715-718 (1991).

${ }^{63}$ Y. B. Gerbig, C. A. Michaels, A. M. Forster, and R. F. Cook, Phys. Rev. B 85, 104102 (2012).

${ }^{64}$ E. Galvani, G. Onida, S. Serra, and G. Benedek, Phys. Rev. Lett. 77, 3573-3576 (1996).

${ }^{65}$ G. Benedek, E. Galvani, S. Sanguinetti, and S. Serra, Chem. Phys. Lett. 244, 339-344 (1995).

${ }^{66}$ M. O'Keeffe, G. B. Adams, and O. F. Sankey, Philos. Mag. Lett. 78, 21-28 (1998).

${ }^{67}$ C. J. Pickard and R. J. Needs, Phys. Rev. B 81, 014106 (2010).

${ }^{68}$ I. H. Lee, J. Lee, Y. J. Oh, S. Kim, and K. J. Chang, Phys. Rev. B 90, 115209 (2014).

${ }^{69}$ H. J. Xiang, B. Huang, E. Kan, S.-H. Wei, and X. G. Gong, Phys. Rev. Lett. 110, 118702 (2013)

${ }^{70}$ Y. Fujimoto, T. Koretsune, S. Saito, T. Miyake, and A. Oshiyama, New J. Phys. 10, 083001 (2008).

${ }^{71}$ R. Hoffmann, A. A. Kabanov, A. A. Golov, and D. M. Proserpio, Angew. Chem., Int. Ed. 55, 10962 (2016).

${ }^{72}$ R. H. Baughman and D. S. Galvao, Chem. Phys. Lett. 211, 110-118 (1993).

${ }^{73}$ B. D. Malone, S. G. Louie, and M. L. Cohen, Phys. Rev. B 81, 115201 (2010).

${ }^{74}$ M. O'Keeffe and N. E. Brese, Acta Crystallogr., Sect. A: Found. Adv. 48, 663-669 (1992).

${ }^{75}$ M. Amsler, J. A. Flores-Livas, L. Lehtovaara, F. Balima, S. A. Ghasemi, D. Machon, S. Pailhes, A. Willand, D. Caliste, S. Botti, A. San Miguel, S. Goedecker, and M. A. L. Marques, Phys. Rev. Lett. 108, 065501 (2012).

${ }^{76}$ S. Goedecker, J. Chem. Phys. 120, $9911-9917$ (2004).

${ }^{77}$ J. Zhai, D. Yu, K. Luo, Q. Wang, Z. Zhao, J. He, and Y. Tian, J. Phys.: Condens. Matter 24, 405803 (2012).

${ }^{78}$ Z. Zhao, B. Xu, X.-F. Zhou, L.-M. Wang, B. Wen, J. He, Z. Liu, H.-T. Wang, and Y. Tian, Phys. Rev. Lett. 107, 215502 (2011).

${ }^{79}$ R. H. Baughman, A. Y. Liu, C. Cui, and P. J. Schields, Synth. Met. 86, 2371 (1997).

${ }^{80}$ A. Bautista-Hernandez, T. Rangel, A. H. Romero, G. M. Rignanese, M. Salazar-Villanueva, and E. Chigo-Anota, J. Appl. Phys. 113, 193504 (2013).

${ }^{81}$ Z. Zhao, F. Tian, X. Dong, Q. Li, Q. Wang, H. Wang, X. Zhong, B. Xu, D. Yu, J. He, H.-T. Wang, Y. Ma, and Y. Tian, J. Am. Chem. Soc. 134, 12362-12365 (2012).

${ }^{82}$ Y. Wang, J. Lv, L. Zhu, and Y. Ma, Phys. Rev. B 82, 094116 (2010).

${ }^{83}$ V. Domnich and Y. Gogotsi, Rev. Adv. Mater. Sci. 3, 1 (2002).

${ }^{84}$ H. Y. Zhao, J. Wang, Q. M. Ma, and Y. Liu, Phys. Chem. Chem. Phys. 15, 17619-17625 (2013)

${ }^{85}$ Q. Q. Wang, B. Xu, J. Sun, H. Y. Liu, Z. S. Zhao, D. L. Yu, C. Z. Fan, and J. L. He, J. Am. Chem. Soc. 136, 9826-9829 (2014).

${ }^{86}$ M. C. Nguyen, X. Zhao, C. Z. Wang, and K. M. Ho, Phys. Rev. B 89, 184112 (2014).

${ }^{87}$ M. O'Keeffe, Acta Crystallogr., Sect. A: Found. Adv. 54, 320 (1998).

${ }^{88}$ O. Delgado-Friedrichs and M. O'Keeffe, Acta Crystallogr., Sect. A: Found. Adv. 61, 358-362 (2005).

${ }^{89}$ Q. Y. Fan, C. C. Chai, Q. Wei, Q. Yang, P. K. Zhou, M. J. Xing, and Y. T. Yang, Mater. Sci. Semicond. Process. 43, 187-195 (2016).

${ }^{90}$ I. H. Lee, Y. J. Oh, S. Kim, J. Lee, and K. J. Chang, Comput. Phys. Commun. 203, 110 (2016).

${ }^{91}$ C. Bonneau and M. O'Keeffe, Inorg. Chem. 54, 808 (2015). 
${ }^{92}$ C. J. Pickard and R. J. Needs, J. Phys.: Condens. Matter 23, 053201 (2011).

${ }^{93}$ J. T. Wang, C. F. Chen, H. Mizuseki, and Y. Kawazoe, Phys. Rev. Lett. 110, 165503 (2013).

${ }^{94}$ B. D. Malone and M. L. Cohen, Phys. Rev. B 85, 024116 (2012).

${ }^{95}$ C. P. Tang, J. Cao, and S. J. Xiong, Physica B 466, 59-63 (2015).

${ }^{96}$ E. A. Belenkov and V. A. Greshnyakov, J. Exp. Theor. Phys. 119, 101-106 (2014).

${ }^{97}$ Q. Y. Fan, C. C. Chai, Q. Wei, H. Y. Yan, Y. B. Zhao, Y. T. Yang, X. H. Yu, Y. Liu, M. J. Xing, J. Q. Zhang, and R. H. Yao, J. Appl. Phys. 118, 185704 (2015).

${ }^{98}$ X. Zhang, Y. Wang, J. Lv, C. Zhu, Q. Li, M. Zhang, Q. Li, and Y. Ma, J. Chem. Phys. 138, 114101 (2013).

${ }^{99}$ Y. G. Guo, Q. Wang, Y. Kawazoe, and P. Jena, Sci. Rep. 5, 14342 (2015)

${ }^{100}$ B. Wen, J. Zhao, M. J. Bucknum, P. Yao, and T. Li, Diamond Relat. Mater. 17, 356 (2008)

${ }^{101}$ S. Froyen, D. M. Wood, and A. Zunger, Phys. Rev. B 36, 4547-4550 (1987).

${ }^{102}$ J. Weber and M. I. Alonso, Phys. Rev. B 40, 5683-5693 (1989).

${ }^{103}$ J. E. Bernard and A. Zunger, Phys. Rev. B 44, 1663-1681 (1991).

${ }^{104}$ K. A. Johnson and N. W. Ashcroft, Phys. Rev. B 54, 14480-14486 (1996).

${ }^{105}$ M. d'Avezac, J.-W. Luo, T. Chanier, and A. Zunger, Phys. Rev. Lett. 108, 027401 (2012)

${ }^{106}$ L. Pizzagalli, EPL 104, 56005 (2013).

${ }^{107}$ Y. J. Oh, I. H. Lee, S. Kim, J. Lee, and K. J. Chang, Sci. Rep. 5, 18086 (2015).

${ }^{108}$ W. C. Oliver and G. M. Pharr, J. Mater. Res. 7, 1564-1583 (1992).

${ }^{109}$ J. S. Field and M. V. Swain, J. Mater. Res. 8, 297-306 (1993).

${ }^{110}$ A. P. Gerk and D. Tabor, Nature 271, 732-733 (1978).

${ }^{111}$ G. M. Pharr, W. C. Oliver, and D. S. Harding, J. Mater. Res. 6, 1129-1130 (1991)

${ }^{112}$ E. R. Weppelmann, J. S. Field, and M. V. Swain, J. Mater. Res. 8, 830-840 (1993).

${ }^{113}$ J. E. Bradby, J. S. Williams, and M. V. Swain, Phys. Rev. B 67, 085205 (2003)

${ }^{114}$ J. E. Bradby, J. S. Williams, J. Wong-Leung, M. V. Swain, and P. Munroe, Appl. Phys. Lett. 77, 3749-3751 (2000).

${ }^{115}$ J. E. Bradby, J. S. Williams, J. Wong-Leung, M. V. Swain, and P. Munroe, J. Mater. Res. 16, 1500-1507 (2001).

${ }^{116}$ A. Kailer, K. G. Nickel, and Y. G. Gogotsi, J. Raman Spectrosc. 30, 939-946 (1999).

${ }^{117}$ I. V. Gridneva, Y. V. Milman, and V. I. Trefilov, Phys. Status Solidi A 14, 177-182 (1972).

${ }^{118}$ D. R. Clarke, M. C. Kroll, P. D. Kirchner, R. F. Cook, and B. J. Hockey, Phys. Rev. Lett. 60, 2156-2159 (1988).

${ }^{119}$ T. Page, W. C. Oliver, and C. J. McHargue, J. Mater. Res. 7, 450 (1992).

${ }^{120}$ A. Kailer, Y. G. Gogotsi, and K. G. Nickel, J. Appl. Phys. 81, 3057-3063 (1997).

${ }^{121}$ V. Domnich, Y. Gogotsi, and S. Dub, Appl. Phys. Lett. 76, 2214-2216 (2000)

${ }^{122}$ S. Wong, B. Haberl, J. S. Williams, and J. E. Bradby, Appl. Phys. Lett. 106, 252103 (2015).

${ }^{123}$ S. Wong, B. Haberl, J. S. Williams, and J. E. Bradby, J. Appl. Phys. 118, 245904 (2015)

${ }^{124}$ S. Ruffell, B. Haberl, S. Koenig, J. E. Bradby, and J. S. Williams, J. Appl. Phys. 105, 093513 (2009).

${ }^{125}$ S. Ruffell, J. E. Bradby, J. S. Williams, and P. Munroe, J. Appl. Phys. 102, 063521 (2007)

${ }^{126}$ B. Haberl, M. Guthrie, G. Shen, J. S. Williams, and J. E. Bradby, "A direct metastable transition from amorphous silicon to the crystalline rhombohedral r8 phase," (unpublished).

${ }^{127}$ B. Haberl, A. C. Y. Liu, J. E. Bradby, S. Ruffell, J. S. Williams, and P. Munroe, Phys. Rev. B 79, 155209 (2009).

${ }^{128}$ E. Holmström, B. Haberl, O. H. Pakarinen, K. Nordlund, F. Djurabekova, R. Arenal, J. S. Williams, J. E. Bradby, T. C. Petersen, and A. C. Y. Liu, J. Non-Cryst. Solids 438, 26-36 (2016).

${ }^{129}$ D. B. Ge, V. Domnich, and Y. Gogotsi, J. Appl. Phys. 95, 2725-2731 (2004)

${ }^{130}$ B. Haberl, M. Guthrie, S. V. Sinogeikin, G. Shen, J. S. Williams, and J. E. Bradby, High Pressure Res. 35, 99-116 (2015).

${ }^{131}$ R. A. Graham, B. Morosin, E. L. Venturini, and M. J. Carr, Annu. Rev. Mater. Sci. 16, 315-341 (1986).
${ }^{132}$ D. Swift, J. Hawreliak, D. Braun, A. Kritcher, S. Glenzer, G. W. Collins, S. Rothman, D. Chapman, and S. Rose, AIP Conf. Proc. 1426, 477 (2012).

${ }^{133}$ R. F. Trunin, Phys.-Usp. 37, 1123-1146 (1994).

${ }^{134}$ P. Loubeyre, P. M. Celliers, D. G. Hicks, E. Henry, A. Dewaele, J. Pasley, J. Eggert, M. Koenig, F. Occelli, K. M. Lee, R. Jeanloz, D. Neely, A. Benuzzi-Mounaix, D. Bradley, M. Bastea, S. Moon, and G. W. Collins, High Pressure Res. 24, 25-31 (2004).

${ }^{135}$ L. V. Al'tshuler, Usp. Fiz. Nauk 85, 197-258 (1965).

${ }^{136}$ M. N. Pavlovskii, Fiz. Tverd. Tela 9, 3192-3197 (1967).

${ }^{137}$ T. Goto, T. Sato, and Y. Syono, Jpn. J. Appl. Phys., Part 2 21, L369-L371 (1982).

${ }^{138}$ S. D. Gilev and A. M. Trubachev, J. Phys.: Condens. Matter 16, 8139-8153 (2004).

${ }^{139}$ H. Kishimura and H. Matsumoto, J. Appl. Phys. 103, 023505 (2008).

${ }^{140}$ P. L. Liu, R. Yen, N. Bloembergen, and R. T. Hodgson, Appl. Phys. Lett. 34, 864-866 (1979).

${ }^{141}$ J. Jia, M. Li, and C. V. Thompson, Appl. Phys. Lett. 84, 3205 (2004).

${ }^{142}$ T. H. R. Crawford, J. Yamanaka, G. A. Botton, and H. K. Haugen, J. Appl. Phys. 103, 053104 (2008).

${ }^{143}$ M. Schade, O. Varlamova, J. Reif, H. Blumtritt, W. Erfurth, and H. S. Leipner, Anal. Bioanal. Chem. 396, 1905-1911 (2010).

${ }^{144}$ M. J. Smith, M.-J. Sher, B. Franta, Y.-T. Lin, E. Mazur, and S. Gradevak, J. Appl. Phys. 112, 083518 (2012).

${ }^{145}$ M. Tsujino, T. Sano, O. Sakata, N. Ozaki, S. Kimura, S. Takeda, M. Okoshi, N. Inoue, R. Kodama, K. F. Kobayashi, and A. Hirose, J. Appl. Phys. 110, 126103 (2011).

${ }^{146}$ Y. Waseda and K. Suzuki, Z. Phys. B: Condens. Matter 20, 339-343 (1975).

${ }^{147}$ P. C. Verburg, L. A. Smillie, G. R. B. E. Römer, B. Haberl, J. E. Bradby, J. S. Williams, and A. J. Huis in't Veld, Appl. Phys. A 120, 683 (2015).

${ }^{148}$ L. Rapp, B. Haberl, J. E. Bradby, E. G. Gamaly, J. S. Williams, and A. V. Rode, Appl. Phys. A 114, 33 (2014).

${ }^{149}$ L. Rapp, B. Haberl, J. E. Bradby, E. G. Gamaly, J. S. Williams, and A. V. Rode, Fundamentals of Laser-Assisted Micro- and Nanotechnologies (Springer, 2014), Vol. 195, pp. 3-26.

${ }^{150}$ F. P. Bundy and J. S. Kasper, Science 139, 340 (1963).

${ }^{151}$ J. D. Joannopoulos and M. L. Cohen, Phys. Rev. B 7, 2644 (1973).

${ }^{152}$ K. Mylvaganam and L. C. Zhang, Comput. Mater. Sci. 81, 10-14 (2014).

${ }^{153}$ K. Mylvaganam and L. C. Zhang, Appl. Phys. A: Mater. Sci. Process. 120, 1391-1398 (2015).

${ }^{154}$ A. Weiss, G. Beil, and H. Meyer, Z. Naturforsch. 34b, 25 (1979).

${ }^{155} \mathrm{M}$. Stutzmann, See article in this issue.

${ }^{156}$ H.-G. Von Schnering, M. Schwarz, and R. Nesper, J. Less-Common Met. 137, 297-310 (1988).

${ }^{157}$ F. Kiefer and T. F. Fassler, Solid State Sci. 13, 636-640 (2011).

${ }^{158}$ F. Kiefer, A. J. Karttunen, M. Doblinger, and T. F. Fassler, Chem. Mater. 23, 4578-4586 (2011).

${ }^{159}$ J. V. Zaikina, E. Muthuswamy, K. I. Lilova, Z. M. Gibbs, M. Zeilinger, G. J. Snyder, T. F. Fassler, A. Navrotsky, and S. M. Kauzlarich, Chem. Mater. 26, 3263-3271 (2014).

${ }^{160}$ M. Zeilinger, L. A. Jantke, L. M. Scherf, F. J. Kiefer, G. Neubuser, L. Kienle, A. J. Karttunen, S. Konar, U. Haussermann, and T. F. Fassler, Chem. Mater. 26, 6603-6612 (2014).

${ }^{161}$ S. Ganguly, N. Kazem, D. Carter, and S. M. Kauzlarich, J. Am. Chem. Soc. 136, 1296-1299 (2014).

${ }^{162}$ J. S. Kasper and S. M. Richards, Acta Crystallogr. 17, 752 (1964).

${ }^{163}$ D. Pan, L. An, Z. Sun, W. Hou, Y. Yang, Z. Yang, and Y. Lu, J. Am. Chem. Soc. 130, 5620 (2008).

${ }^{164}$ S. Bobev and S. C. Sevov, J. Solid State Chem. 153, 92-105 (2000).

${ }^{165}$ A. Ker, E. Todorov, R. Rousseau, K. Uehara, F. X. Lannuzel, and J. S. Tse, Chem. - Eur. J. 8, 2787-2798 (2002).

${ }^{166}$ M. Beekman and G. S. Nolas, J. Mater. Chem. 18, 842-851 (2008).

${ }^{167}$ C. Cros and M. Pouchard, C. R. Chim. 12, 1014-1056 (2009).

${ }^{168}$ S. Yamanaka, Dalton Trans. 39, 1901-1915 (2010).

${ }^{169}$ C. Cros, M. Pouchard, and P. Hagenmuller, C. R. Acad. Sci. 260, 4764 (1965).

${ }^{170}$ H. Morito, T. Yamada, T. Ikeda, and H. Yamane, J. Alloys Compd. 480, 723-726 (2009).

${ }^{171}$ O. O. Kurakevych, T. A. Strobel, D. Y. Kim, T. Muramatsu, and V. V. Struzhkin, Cryst. Growth Des. 13, 303-307 (2013).

${ }^{172}$ S. Yamanaka, M. Komatsu, M. Tanaka, H. Sawa, and K. Inumaru, J. Am. Chem. Soc. 136, 7717 (2014)

${ }^{173}$ P. T. Hutchins, O. Leynaud, L. A. O’Dell, M. E. Smith, P. Barnes, and P. F. McMillan, Chem. Mater. 23, 5160-5167 (2011). 
${ }^{174}$ C. Cros, M. Pouchard, and P. Hagenmuller, J. Solid State Chem. 2, 570 (1970).

${ }^{175}$ B. Böhme, A. Guloy, Z. Tang, W. Schnelle, U. Burkhardt, M. Baitinger, and Y. Grin, J. Am. Chem. Soc. 129, 5348-5349 (2007).

${ }^{176}$ A. Ammar, C. Cros, M. Pouchard, N. Jaussaud, J. M. Bassat, G. Villeneuve, M. Duttine, M. Menetrier, and E. Reny, Solid State Sci. 6, 393 (2004).

${ }^{177}$ S. Stefanoski, H. Liu, Y. Yao, and T. A. Strobel, Inorg. Chem. 54, 10761 (2015).

${ }^{178}$ W. Shockley and H. J. Queisser, J. Appl. Phys. 32, 510 (1961).

${ }^{179}$ R. A. Street, Hydrogenated Amorphous Silicon (Cambridge University Press, Cambridge, 1991).

${ }^{180}$ L. Krishna, L. L. Baranowski, A. D. Martinez, C. A. Koh, C. P. Taylor, A. C. Tamboli, and E. S. Toberer, CrystEngComm 16, 3940-3949 (2014).

${ }^{181}$ L. Krishna and C. A. Koh, MRS Energy Sustainability 2, E8 (2015).

${ }^{182}$ A. M. Guloy, R. Ramlau, Z. J. Tang, W. Schnelle, M. Baitinger, and Y. Grin, Nature 443, 320-323 (2006).

${ }^{183}$ F. B. Bundy, H. T. Hall, H. M. Strong, and R. J. Wentorf, Jr., Nature 176, 51 (1955).

${ }^{184}$ M. P. Bovenkerk, F. P. Bundy, H. T. Hall, H. M. Strong, and R. H. Wentorf, Jr., Nature 184, 1094 (1959).

${ }^{185}$ R. H. Wentorf, Jr., J. Chem. Phys. 34, 809 (1961).

${ }^{186}$ M. I. Eremets, A. G. Gavriliuk, I. A. Trojan, D. A. Dzivenko, and R. Boehler, Nat. Mater. 3, 558 (2004)

${ }^{187}$ A. Zerr, G. Miehe, G. Serghiou, M. Schwarz, E. Kroke, R. Riedel, H. Fuess, P. Kroll, and R. Boehler, Nature 400, 340-342 (1999).

${ }^{188}$ G. Serghiou, G. Miehe, O. Tschauner, A. Zerr, and R. Boehler, J. Chem. Phys. 111, 4659 (1999).

${ }^{189}$ E. Horvath-Bordon, R. Riedel, P. F. McMillan, P. Kroll, G. Miehe, P. A. van Aken, A. Zerr, P. Hoppe, O. Shebanova, I. McLaren, S. Lauterbach, E. Kroke, and R. Boehler, Angew. Chem., Int. Ed. 46, 1476 (2007)

${ }^{190}$ V. L. Solozhenko and E. Gregoryanz, Mater. Today 8, 44 (2005).
${ }^{191}$ R. Boehler, M. Guthrie, J. J. Molaison, A. M. dos Santos, S. V. Sinogeikin, S. Machida, N. Pradhan, and C. A. Tulk, High Pressure Res. 33, 546 (2013).

${ }^{192}$ S. Klotz, Techniques in High Pressure Neutron Scattering (CRC Press, Taylor \& Francis Group, Boca Raton, FL, 2012).

${ }^{193}$ N. Kawai and S. Endo, Rev. Sci. Instrum. 41, 1178-1181 (1970).

${ }^{194}$ R. Abbaschian, H. Zhu, and C. Clarke, Diamond Relat. Mater. 14, 1916-1919 (2005).

${ }^{195}$ L. Vel, G. Demazeau, and J. Etourneau, Mater. Sci. Eng., B 10, 149-164 (1991).

${ }^{196}$ R. Rao, J. E. Bradby, and J. S. Williams, Appl. Phys. Lett. 91, 123113 (2007).

${ }^{197}$ W. Steen and J. Mazumder, Laser Material Processing, 4th ed. (SpringerVerlag, London, 2010).

${ }^{198}$ J. C. Angus and C. C. Hayman, Science 241, 913-921 (1988).

${ }^{199}$ K. E. Spear and J. P. Dismukes, Synthetic Diamond: Emerging CVD Science and Technology (John Wiley \& Sons, 1994), Vol. 25.

${ }^{200}$ H. I. T. Hauge, M. A. Verheijen, S. Conesa-Boj, T. Etzelstorfer, M. Watzinger, D. Kriegner, I. Zardo, C. Fasolato, F. Capitani, P. Postorino, S. Kolling, A. Li, S. Assali, J. Stangl, and E. Bakkers, Nano Lett. 15, 5855-5860 (2015).

${ }^{201}$ P. B. Mirkarimi, K. F. McCarty, and D. L. Medlin, Mater. Sci. Eng., R 21, 47-100 (1997).

${ }^{202}$ F. P. Bundy and J. S. Kasper, J. Chem. Phys. 46, 3437 (1967).

${ }^{203}$ S. J. Clark, G. J. Ackland, and J. Crain, Phys. Rev. B 52, 15035 (1995).

${ }^{204}$ F. P. Bundy, J. Chem. Phys. 38, 631 (1963).

${ }^{205}$ R. J. Nelmes, M. I. McMahon, N. G. Wright, D. R. Allan, and J. S. Loveday, Phys. Rev. B 48, 9883 (1993).

${ }^{206}$ B. Haberl, M. Guthrie, B. D. Malone, J. S. Smith, S. V. Sinogeikin, M. L. Cohen, J. S. Williams, G. Shen, and J. E. Bradby, Phys. Rev. B 89, 144111 (2014).

${ }^{207}$ B. C. Johnson, B. Haberl, S. Deshmukh, B. D. Malone, M. L. Cohen, J. C. McCallum, J. S. Williams, and J. E. Bradby, Phys. Rev. Lett. 110, 085502 (2013). 